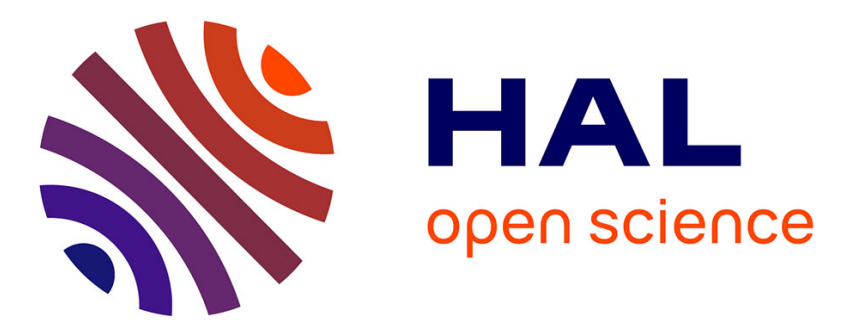

\title{
Forward and backward motion control of a vibro-impact capsule system
}

\author{
Yang Liu, Ekaterina Pavlovskaia, Marian Wiercigroch, Zhike Peng
}

\section{To cite this version:}

Yang Liu, Ekaterina Pavlovskaia, Marian Wiercigroch, Zhike Peng. Forward and backward motion control of a vibro-impact capsule system. International Journal of Non-Linear Mechanics, 2015, 70, pp.30 - 46. 10.1016/j.ijnonlinmec.2014.10.009 . hal-01510830

\section{HAL Id: hal-01510830 \\ https://hal.science/hal-01510830}

Submitted on 20 Apr 2017

HAL is a multi-disciplinary open access archive for the deposit and dissemination of scientific research documents, whether they are published or not. The documents may come from teaching and research institutions in France or abroad, or from public or private research centers.
L'archive ouverte pluridisciplinaire HAL, est destinée au dépôt et à la diffusion de documents scientifiques de niveau recherche, publiés ou non, émanant des établissements d'enseignement et de recherche français ou étrangers, des laboratoires publics ou privés. 


\title{
Forward and backward motion control of a vibro-impact capsule system
}

\author{
Yang Liu ${ }^{a}$, Ekaterina Pavlovskaia ${ }^{\mathrm{b}}$, Marian Wiercigroch ${ }^{\mathrm{b}}$, Zhike Peng ${ }^{\mathrm{c}}$ \\ a School of Engineering, Robert Gordon University, Garthdee Road, Aberdeen AB10 7GJ, UK \\ ${ }^{\mathrm{b}}$ Centre for Applied Dynamics Research, School of Engineering, King's College, University of Aberdeen, Aberdeen AB24 $3 U E$, UK \\ ' State Key Laboratory of Mechanical System and Vibration, Shanghai Jiao Tong University, Shanghai 200240, PR China
}

\begin{abstract}
A capsule system driven by a harmonic force applied to its inner mass is considered in this study. Four various friction models are employed to describe motion of the capsule in different environments taking into account Coulomb friction, viscous damping, Stribeck effect, pre-sliding, and frictional memory. The non-linear dynamics analysis has been conducted to identify the optimal amplitude and frequency of the applied force in order to achieve the motion in the required direction and to maximize its speed. In addition, a position feedback control method suitable for dealing with chaos control and coexisting attractors is applied for enhancing the desirable forward and backward capsule motion. The evolution of basins of attraction under control gain variation is presented and it is shown that the basin of the desired attractors could be significantly enlarged by slight adjustment of the control gain improving the probability of reaching such an attractor.
\end{abstract}

\author{
Keywords: \\ Capsule \\ Vibro-impact \\ Friction \\ Motion control \\ Position feedback control
}

\section{Introduction}

In the last few years, there was a growing interest in developing mobile mechanisms for minimally invasive surgical operation [1-4] and engineering pipeline inspection [5-7]. Particularly, investigation of a capsule system moving under internal force when overcoming environmental resistance has attracted significant attention, e.g. [8-11]. The merit of such a system is its simplicity in mechanical design and control which does not require any external driving mechanisms while allows it to move independently in a complex environment unaccessible to the legged and wheeled mechanisms [12,13]. However, any small uncertainties in friction or system parameters may lead to qualitative change of the dynamics of the capsule system [14]. Therefore understanding of the dynamics and motion control under different frictional environments for such a system is essential.

This paper studies the vibro-impact dynamics of a capsule system in the environments described by four friction models under variation of the amplitude and the frequency of harmonic excitation. The physical model of the vibro-impact capsule system is shown in Fig. 1 which consists of a capsule main body interacting with an internal harmonically driven mass. An initial bifurcation study of this system was carried out in [11]. The study has shown that the dynamic behaviour of the system is mainly periodic, and the best progression can be achieved through a careful choice of system parameters, such as mass ratio, stiffness ratio, amplitude and frequency of excitation. The dynamics of the capsule system in various frictional environments under variation of the mass ratio was investigated in [14] which suggested that directional control of the system can be achieved either by varying its mass ratio or by switching between coexisting attractors. This paper proposes a position feedback control law in order to control the capsule moving along a desired direction. Additionally, we also show that our proposed control method is capable for the control of chaos or coexisting attractors for ensuring an efficient performance of the system.

Control of vibro-impact systems has attracted great attention for many years, e.g. [15-20]. In [15], control of a double impacting oscillator using displacement feedback was studied, and the effect of how grazing impacts limit the stability regions of certain periodic orbits was discussed. To retain the existence of a desired attractor near the grazing trajectory, Dankowicz and Jerrelind [16] employed a discrete linear feedback control strategy. In [17], Souza and Caldas introduced a transcendental map to determine the value of parameter perturbation for controlling the vibro-impact systems which exhibited desired unstable periodic orbit embedded in a chaotic attractor. Lee and Yan studied the control algorithms for position control of an impact oscillator and synchronization of two impact 


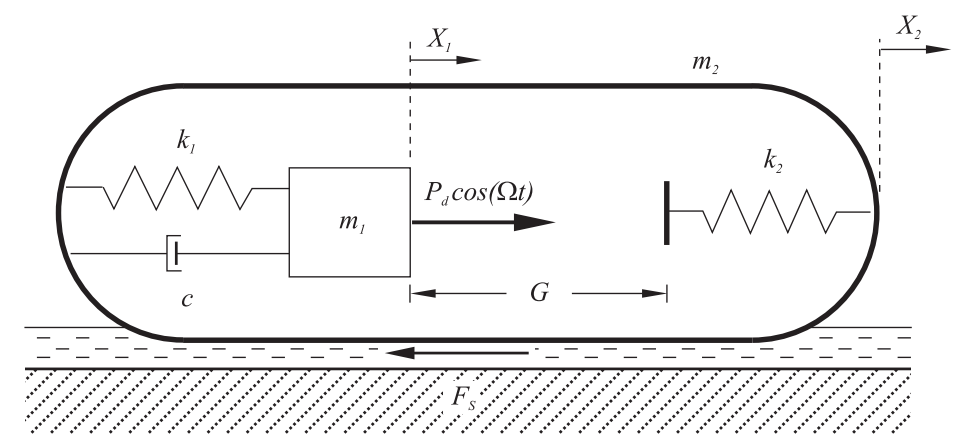

Fig. 1. Physical model of the vibro-impact capsule system.

oscillators in [18]. A feedback control technique using a smallamplitude damping signal was studied in [19] for suppressing chaotic behaviour of an impact oscillator. Later on, Wang et al. [20] developed an impulsive control method to stabilize the chaotic motion for a class of vibro-impact systems. In [21], Liu et al. proposed an intermittent control method for a class of non-autonomous dynamics systems that naturally exhibited coexisting attractors, and demonstrated its applicability to an impact oscillator numerically and experimentally. Basically, vibro-impact systems mainly fall into two categories: the impact oscillator with fixed impact body, e.g. $[22,23]$ and the impact oscillator with one-side drifting impact body e.g. [24-26]. However this paper studies the control of a vibroimpact system with impact body drifting forward and backward which has never been considered in the literature before. Compared to the impact oscillator with fixed impact body, this structure induces more complicated dynamics when experiences various environmental resistance. Both optimization and directional control issues must be considered for our proposed system, while only optimization is needed for the impact oscillator with one-side drifting impact body. This paper also uses basins of attraction for the first time to investigate the possibility of switching between coexisting attractors by using the proposed control method.

The rest of this paper is organized as follows. In Section 2, mathematical modelling of the vibro-impact capsule system is presented, and the four different friction models used in this paper are briefly introduced. In Section 3, a non-linear dynamic analysis of the capsule system is conducted by varying the amplitude of excitation. In Section 4, influence of excitation frequency on capsule dynamics is investigated, and its global and local optima are studied. In Section 5, forward and backward motion control of the capsule system is studied by using a position feedback control law. Here the capabilities of the proposed control method for the control of chaos and coexisting attractors are demonstrated through extensive numerical studies. Finally, some concluding remarks are drawn in Section 6.

\section{Mathematical modelling}

\subsection{Equations of motion}

This work considers a two degrees-of-freedom dynamical system depicted in Fig. 1, where a movable internal mass $m_{1}$ is driven by a harmonic force with amplitude $P_{d}$ and frequency $\Omega$ interacting with a rigid capsule $m_{2}$ via a linear spring with stiffness $k_{1}$ and a viscous damper with damping coefficient $c . X_{1}$ and $X_{2}$ represent the absolute displacements of the internal mass and the capsule, respectively. The internal mass contacts a weightless plate connected to the capsule by a secondary linear spring with stiffness $k_{2}$ when the relative displacement $X_{1}-X_{2}$ is larger or equals to the gap $G$. When the force acting on the capsule exceeds the threshold of the dry friction force $F_{b}$ between the capsule and the supporting environmental surface, the bidirectional motion of the capsule occurs, and the friction force $F_{S}$ is applied to the capsule.

To simplify the analysis, we introduce the following nondimensional variables:

$\tau=\Omega_{0} t, \quad x_{i}=\frac{k_{1}}{P_{f}} X_{i}, \quad y_{i}=\frac{d x_{i}}{d \tau}=\frac{k_{1}}{\Omega_{0} P_{f}} \dot{X}_{i}, \quad \dot{y}_{i}=\frac{d y_{i}}{d \tau}=\frac{k_{1}}{\Omega_{0}^{2} P_{f}} \ddot{X}_{i}$,

$f_{s}=\frac{F_{s}}{P_{f}}, \quad f_{b}=\frac{F_{b}}{P_{f}}$,

and parameters

$\Omega_{0}=\sqrt{\frac{k_{1}}{m_{1}}}, \quad \omega=\frac{\Omega}{\Omega_{0}}, \quad \alpha=\frac{P_{d}}{P_{f}}, \quad \xi=\frac{c}{2 m_{1} \Omega_{0}}, \quad \delta=\frac{k_{1}}{P_{f}} G, \quad \beta=\frac{k_{2}}{k_{1}}$, $\gamma=\frac{m_{2}}{m_{1}}$

where $i=1,2$, and $P_{f}$ is the threshold of Coulomb friction. The considered system operates in bidirectional stick-slip phases which contain the following modes: stationary capsule without contact, moving capsule without contact, stationary capsule with contact, moving capsule with contact. A detailed consideration of these modes and dimensional form of the equations of motion can be found in [11]. The comprehensive equations of motion for the vibro-impact capsule system are written as

$\dot{x}_{1}=y_{1}$,

$\dot{y}_{1}=\alpha \cos (\omega \tau)+\left(x_{2}-x_{1}\right)+2 \xi\left(y_{2}-y_{1}\right)-H_{3} \beta\left(x_{1}-x_{2}-\delta\right)$,

$\dot{x}_{2}=y_{2}\left(H_{1}\left(1-H_{3}\right)+H_{2} H_{3}\right)$,

$\dot{y}_{2}=\left(H_{1}\left(1-H_{3}\right)+H_{2} H_{3}\right)\left(-f_{s}-\left(x_{2}-x_{1}\right)-2 \xi\left(y_{2}-y_{1}\right)\right.$

$$
\left.+H_{3} \beta\left(x_{1}-x_{2}-\delta\right)\right) / \gamma \text {, }
$$

where $H(\cdot)$ is the Heaviside function and functions $H_{i}(i=1,2,3)$ are defined as

$H_{1}=H\left(\left|\left(x_{2}-x_{1}\right)+2 \xi\left(y_{2}-y_{1}\right)\right|-f_{b}\right)$,

$H_{2}=H\left(\left|\left(x_{2}-x_{1}\right)+2 \xi\left(y_{2}-y_{1}\right)-\beta\left(x_{1}-x_{2}-\delta\right)\right|-f_{b}\right)$,

$H_{3}=H\left(x_{1}-x_{2}-\delta\right)$.

\subsection{Friction models}

In [14], the environmental resistance was described by four different friction models given in Table 1 which took into account Coulomb friction, viscous damping, Stribeck effect, pre-sliding, and frictional memory. As it is known, the Coulomb friction model provides the first approximation of dry frictional contact, and the Coulomb viscous damping model takes into account the viscosity of lubricated contact. Both Coulomb Stribeck and seven-parameter models [27] describe the friction of thicker lubricated contact, while the later one can comprehensively interpret the resistant force at a very low relative speed. The work in [14] has revealed that when the weight of the internal mass is smaller than the weight of the capsule, the nature of the friction mechanism 
becomes less significant as it does not influence so much the capsule dynamics. This paper further investigates the capsule dynamics under variation of the amplitude and frequency of external excitation using these four friction models, and proposes a control method which can switch the system state from chaotic to periodic motion or from a current attractor to a desired one.

\section{Influence of excitation amplitude}

This section compares capsule dynamic responses with dry, lubricated, and thicker lubricated contact surfaces, and investigates the influence of amplitude of excitation on capsule motion. The comparison was carried out using the bifurcation diagrams as shown in Fig. 2 where the velocity $y_{1}^{*}$, which is a projection of the Poincare map on the $y_{1}$-axis, was plotted as a function of the amplitude of excitation for the systems with (a) the Coulomb, (b) the Coulomb viscous damping, (c) the Coulomb Stribeck, and (d) the seven-parameter models. The main attractors of the system are shown by black dots and the coexisting attractors are marked by red dots. The calculations were run for 300 cycles of the external excitation, and the data for the first 200 cycles were omitted to ensure the steady state response, whereas the next 100 values of the velocity $y_{1}^{*}$ were plotted in the bifurcation diagrams for each value of the amplitude of excitation. For simplicity, we use abbreviations in bifurcation diagram to describe periodic motion of the system, e.g. P-1-2 represents a periodic-1 motion with two impacts per period of external excitation.

\subsection{Periodic motion}

As can be seen from Fig. 2(a), when the system has dry contact surface, periodic motion of the capsule is observed for $\alpha \in[0.1,0.132)$ and $\alpha \in[0.928,2.0]$. When the system has lubricated contact surface, similar periodic motion is found for $\alpha \in[0.1,0.132)$ and $\alpha \in[0.942,2.0]$ in Fig. 2(b). When the lubrication becomes thicker, periodic motion is recorded for $\alpha \in[0.1,0.228), \alpha \in[0.572,0.84)$, and $\alpha \in[1.65,2.0]$ in Fig. 2(c) for the system with the Coulomb Stribeck model, and $\alpha \in[0.1,0.228), \alpha \in[0.724,0.914)$, and $\alpha \in[1.722,2.0]$ in Fig. 2(d) for the system with the seven-parameter model. Our bifurcation study reveals that the system has similar dynamic response when its contact surface is dry and thinly lubricated, and periodic motion of the system can be obtained when the amplitude of the harmonic force $P_{d}$ is greater than the threshold of friction $P_{f}(\mathrm{i}$. e. $\alpha>1$ ). When the capsule has thicker lubricated contact, capsule motion becomes more complicated and the range of excitation amplitude for periodic motion is quite narrow. Therefore forward and backward motion control of the capsule system through adjusting excitation amplitude is limited.

Comparing the periodic motion in $\alpha \in[0.1,0.132)$ for the systems with the Coulomb and the Coulomb viscous damping models with the periodic motion in $\alpha \in[0.1,0.228)$ for the systems with the

Table 1

Friction models [14].

\begin{tabular}{|c|c|c|c|c|}
\hline Friction model & Description & Static friction & Dynamic friction $f_{s}$ & Threshold $f_{b}$ \\
\hline Coulomb & Dry contact & 0 & $\operatorname{sign}\left(\dot{x}_{2}\right)$ & 1 \\
\hline Coulomb viscous damping & Lubricated contact & 0 & $\operatorname{sign}\left(\dot{x}_{2}\right)+\mu_{v} \dot{x}_{2}$ & 1 \\
\hline Coulomb Stribeck & Thicker lubricated contact & 0 & $\operatorname{sign}\left(\dot{x}_{2}\right)+\operatorname{sign}\left(\dot{x}_{2}\right) e^{-\left|\dot{x}_{2}\right| / v_{s}}$ & 2 \\
\hline Seven-parameter & Lubricated contact at very low relative speed & $k_{s} x_{2}$ & $\operatorname{sign}\left(\dot{x}_{2}\right)+\mu_{v} \dot{x}_{2}+\frac{\operatorname{sign}\left(\dot{x}_{2}\right)}{1+\left(\frac{\dot{x}_{2}}{v_{s}}\left(\tau-\tau_{d}\right.\right.}$ & 2 \\
\hline
\end{tabular}

(a)

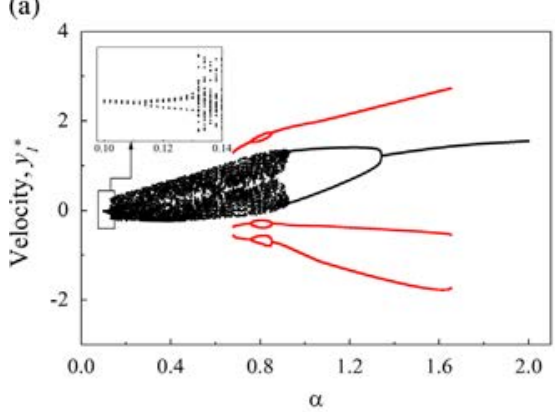

(c)

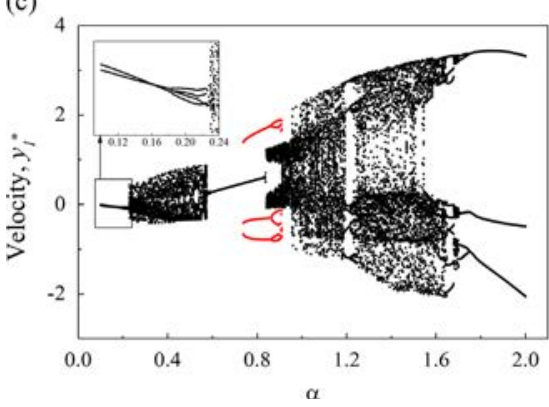

(b)

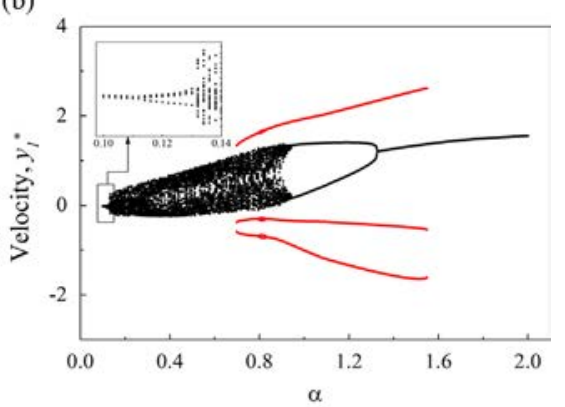

(d)

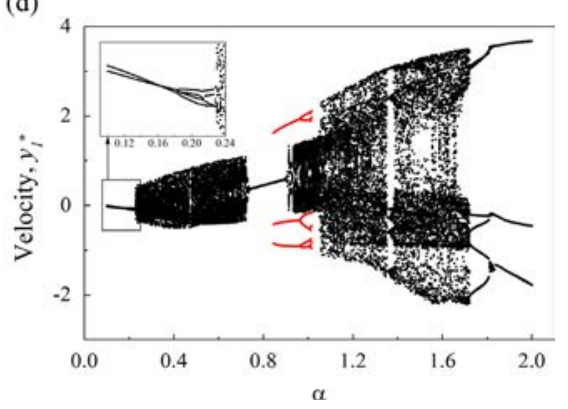

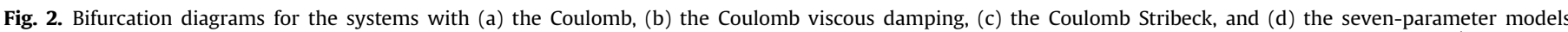

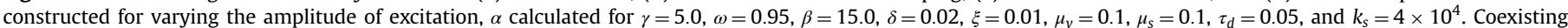
attractors are shown by red dots. (For interpretation of the references to colour in this figure caption, the reader is referred to the web version of this paper.) 
Coulomb Stribeck and the seven-parameter models, it is noted that the periodic motion with thicker lubricated contact exists for a broader range of excitation amplitude than the one with dry and thinly lubricated contacts. The reason for such a difference is caused by the threshold of friction model which dominates the capsule motion at a very low absolute speed as illustrated in Fig. 3. As can be seen in Fig. 3(a) and (d), the system with the Coulomb model has a similar period- 4 motion with the one with the Coulomb viscous damping model at $\alpha=0.13$. Both capsules have small forward progression, although minor difference can be observed between the displacements in Fig. 3(b) and (e) which is due to the dependence of the capsule velocity for the Coulomb viscous damping friction as shown in Fig. 3(f). Similar period-4 motions are observed at $\alpha=0.20$ for the systems with the Coulomb Stribeck and the seven-parameter models as shown in Fig. 3(g)-(i) and (j)-(l), where similar mass motion but no capsule progression can be observed. The comparison of Fig. 3(i) and (1) indicates that the elastic force acting on the capsule does not overcome the threshold of friction but the seven-parameter model can better interpret the friction force between two sticking surfaces.
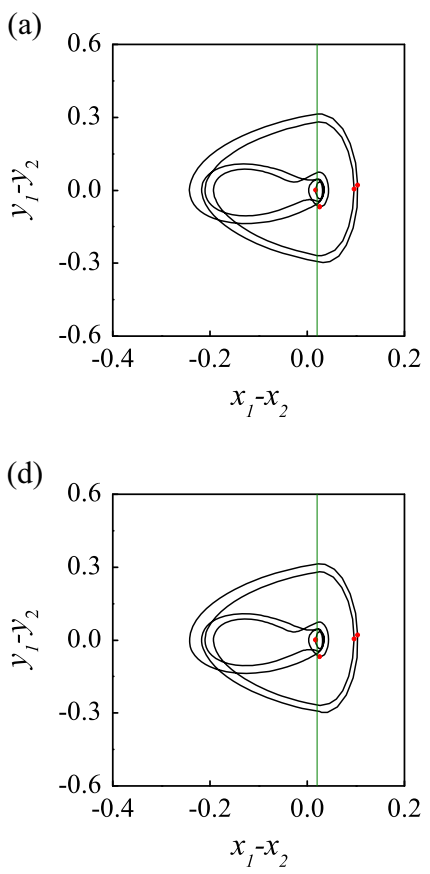

(g)

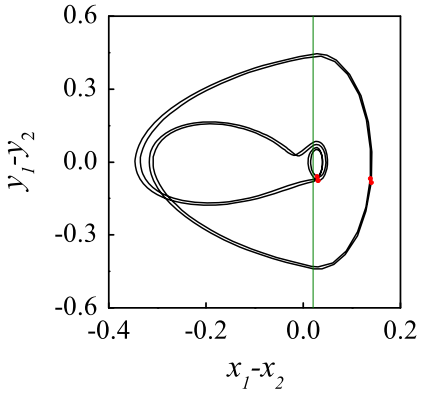

(j)

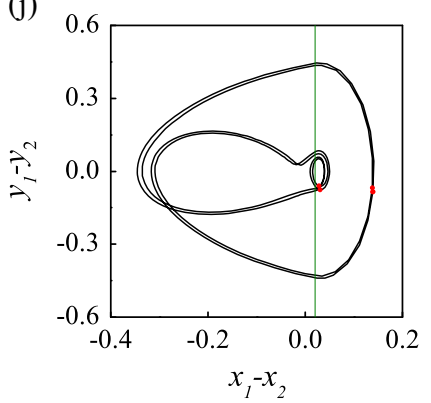

(b)

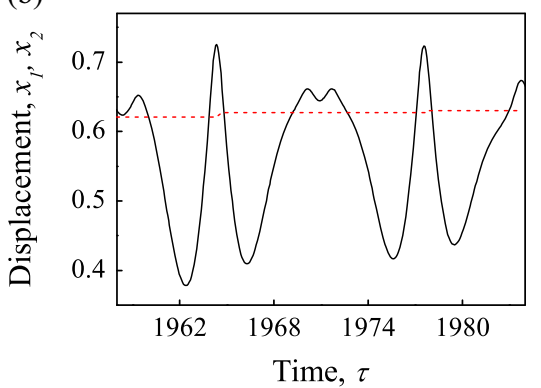

(e)

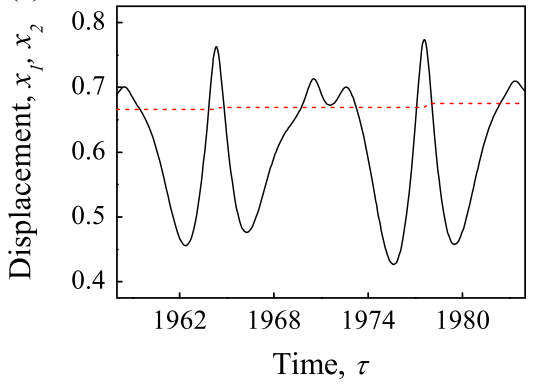

(h)

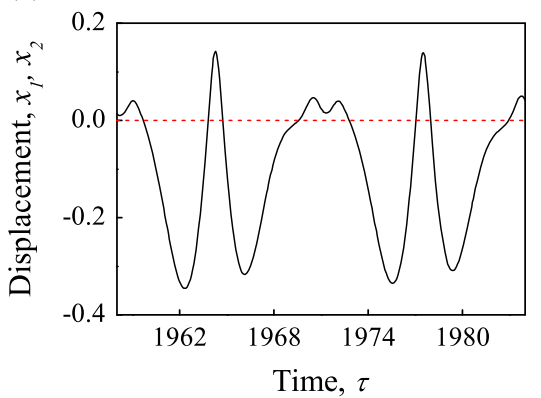

(k)

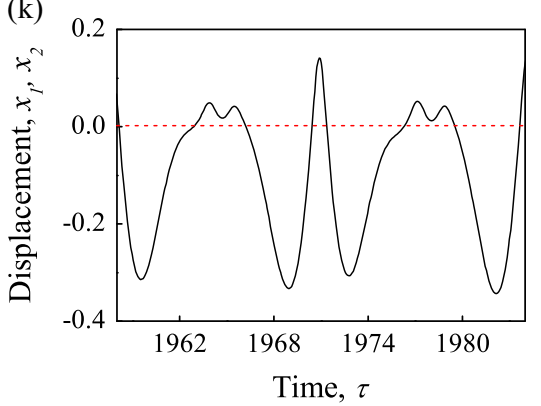

(c)

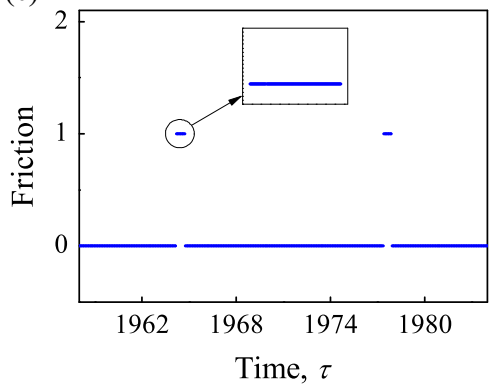

(f)

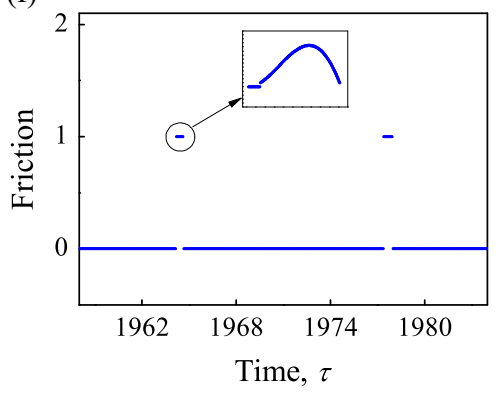

(i)

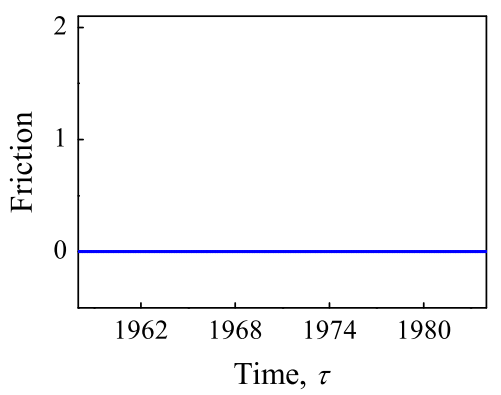

(1)

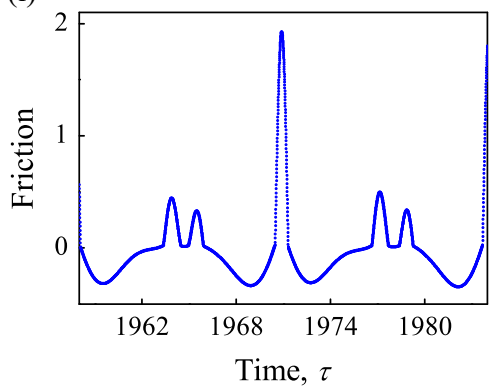

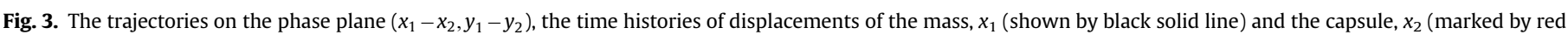

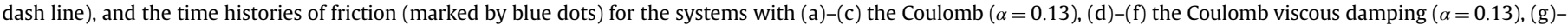

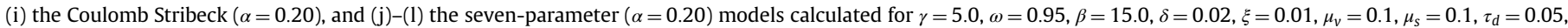

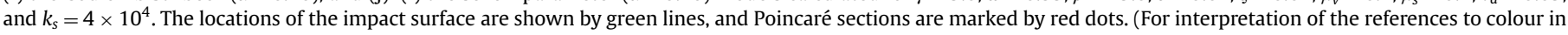
this figure caption, the reader is referred to the web version of this paper.) 


\subsection{Grazing induced chaotic motion}

When amplitude of excitation is $\alpha=0.802$, the chaotic motions for the Coulomb and the Coulomb viscous damping models are compared with the period- 1 motions for the Coulomb Stribeck and the seven-parameter models in Fig. 4. As can be seen in Fig. 4(b) and (e), the time histories of the mass and the capsule displacements are shown which indicate the grazing induced chaotic response where the mass just contacts with the secondary spring of the capsule. When the elastic force acting on the capsule is larger than the threshold of the friction, backward motion of the capsule is observed. For the displacements of the systems with the Coulomb Stribeck and the seven-parameter models, only forward period-1 motions are observed in Fig. 4(h) and (k). In this case, the speed of the capsule with thicker lubricated contact is much faster than the speed with dry and thinly lubricated contacts although its frictional threshold is larger than the other two cases. This observation reveals that at some situations, larger friction may help to retain system stability and keeps system response in an optimal regime.

\subsection{Evolution of chaotic motion}

As can be seen from Fig. 2(c) and (d), the chaotic motions for the systems with the Coulomb Stribeck and the seven-parameter models (a)

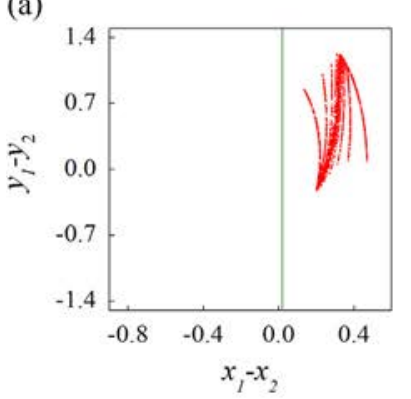

(d)

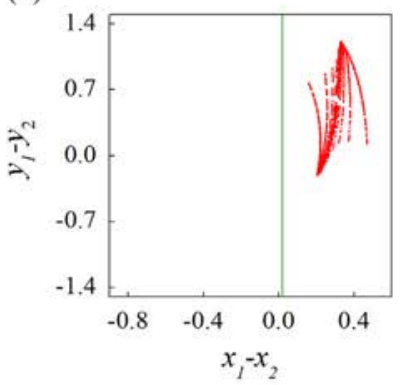

(g)

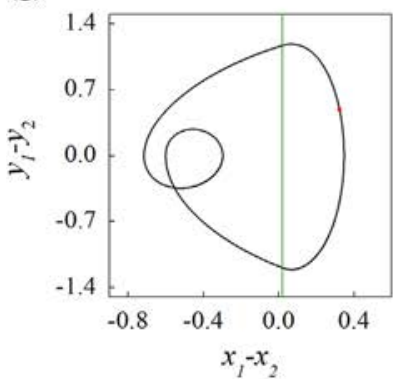

(j)

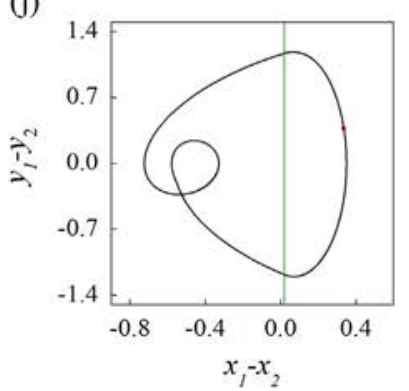

(b)

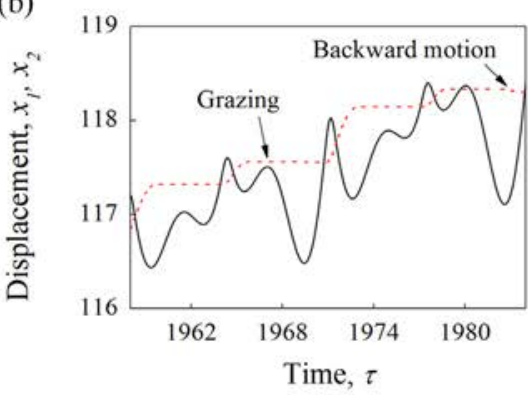

(e)

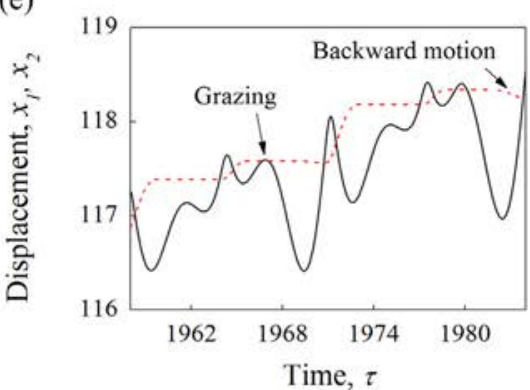

(h)

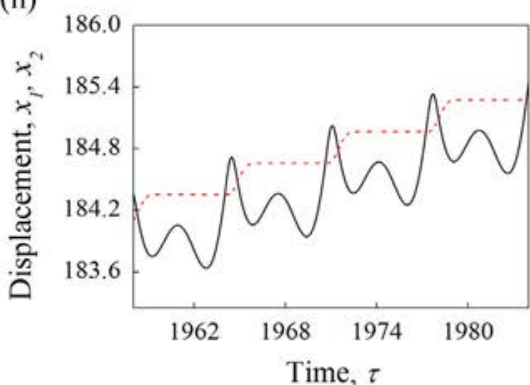

(k)

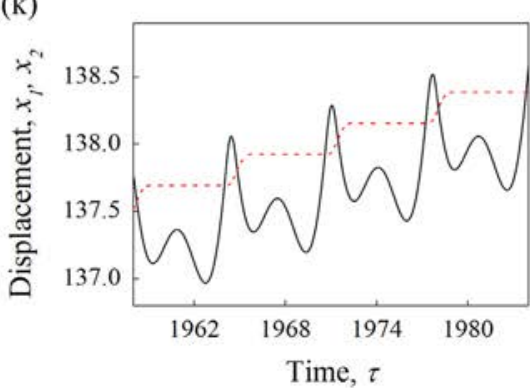

(c)

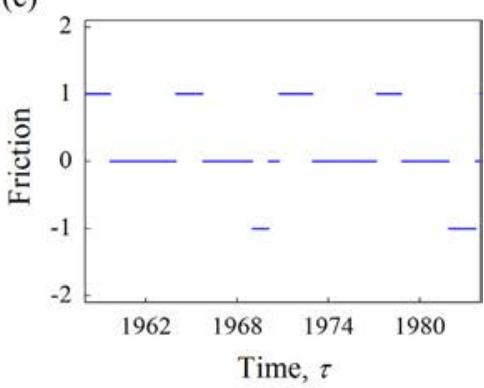

(f)

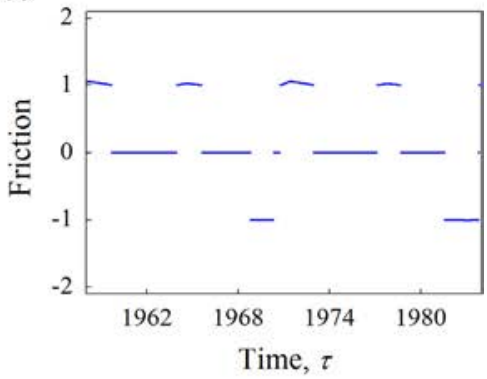

(i)

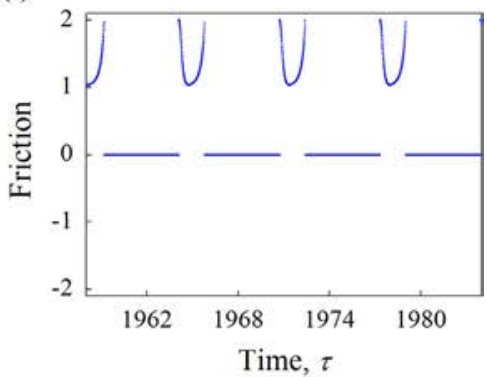

(1)

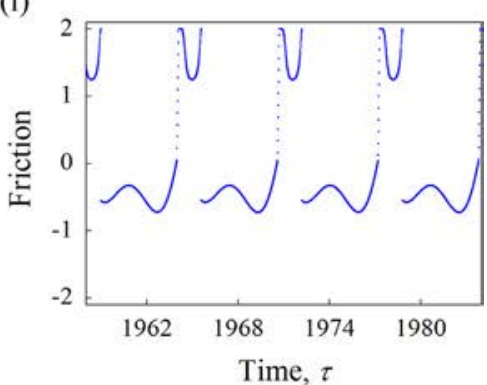

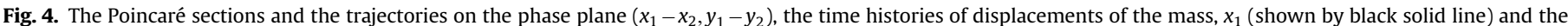

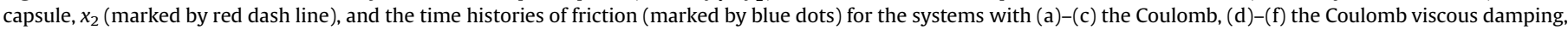

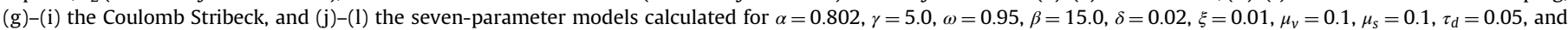

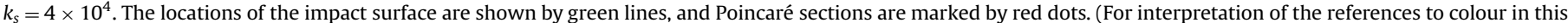
figure caption, the reader is referred to the web version of this paper.) 
are recorded for $\alpha \in[0.84,1.65)$ and $\alpha \in[0.914,1.722)$, respectively. As the amplitude of excitation increases, both chaotic motions are settled down at a period-3 motion with five impacts via a reverse period doubling cascade at $\alpha=1.65$ for the Coulomb Stribeck model and $\alpha=1.722$ for the seven-parameter model. Poincaré maps for both chaotic motions using different values of amplitude of excitation are shown in Fig. 5 which presents two series of strange attractors. The development of the strange attractor for the system with the Coulomb Stribeck model is shown in Fig. 5(a)-(d), and the development of another one for the system with the sevenparameter model is given in Fig. 5(e)-(h). As can be seen from these figures, the larger the amplitude of excitation, the less complex the behaviour of the system. Fig. 6 depicts a special bifurcation for the system with thicker lubricated contact which is the transition of the capsule motion from forward only to forward and backward. It can be seen from Fig. 6, as the amplitude of excitation increases from $\alpha=1.1$ to $\alpha=1.3$, the backward motion of the capsule is recorded. As the amplitude of excitation increases, the best progression for the system with the Coulomb Stribeck model is achieved by a period-3 motion with five impacts per period at $\alpha=1.762$, while the best progression for the system with the sevenparameter model is achieved at $\alpha=1.882$.

(a)

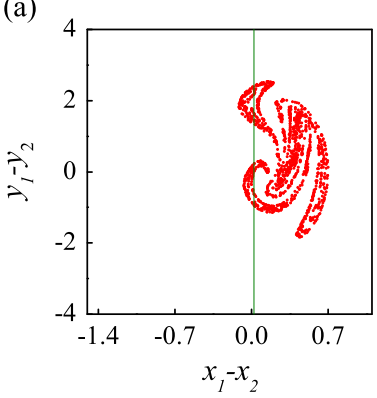

(e)

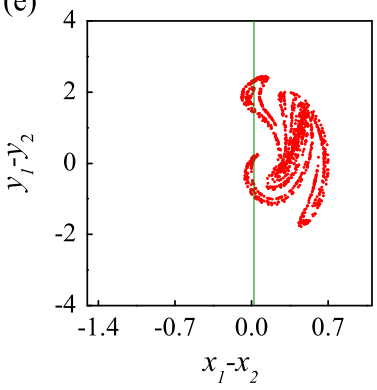

(b)

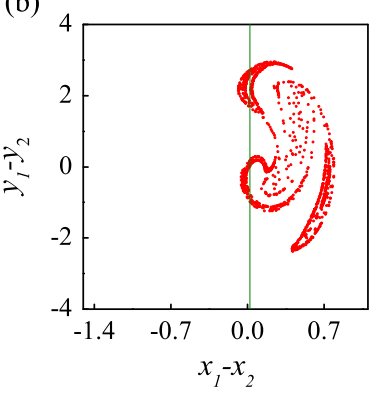

(f)

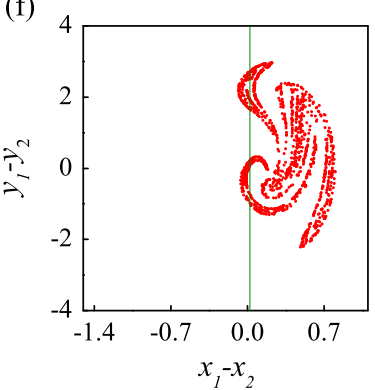

\subsection{Average progression of the capsule}

The speed of capsule progression is a critical performance index for monitoring the system under the optimal regime of control parameters. Fig. 7 shows the calculation of the average progression of the capsule per period of external excitation for the systems with the four friction models. As can be seen in Fig. 7(a) and (b), the average progressions for the systems with the Coulomb and the Coulomb viscous damping models increase when their amplitudes of excitation increase. For the systems with the Coulomb Stribeck and the seven-parameter models in Fig. 7(c) and (d), the fastest average progression is recorded for a period-3 motion with five impacts which has been introduced in Fig. 6. The average progression made by coexisting attractor is marked by red dots in Fig. 7 where no significant improvement on capsule average progression is obtained. For the systems with the Coulomb and the Coulomb viscous damping models shown in Fig. 7(a) and (b), the average progression made by the coexisting period-3 motion is even smaller than the one made by the main period-2 attractor. Therefore in this situation coexisting attractor should be avoided, and the system should be controlled within its main attractor. (c)

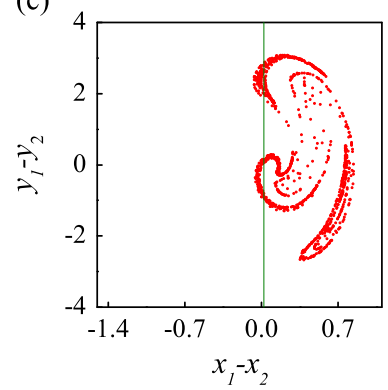

(g)

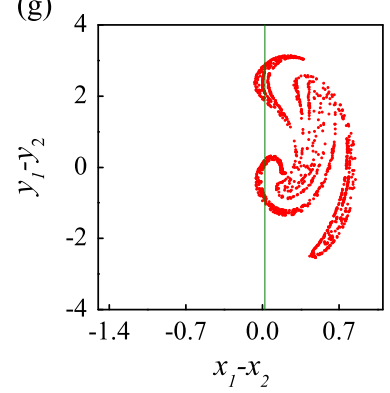

(d)

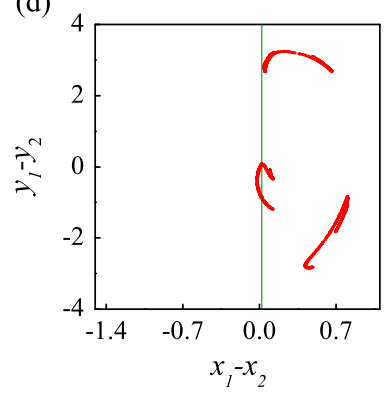

(h)

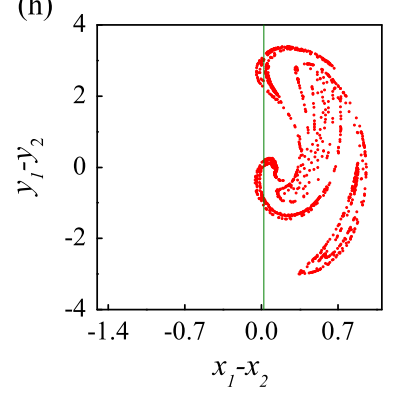

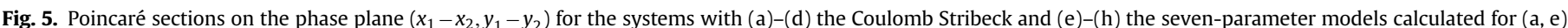

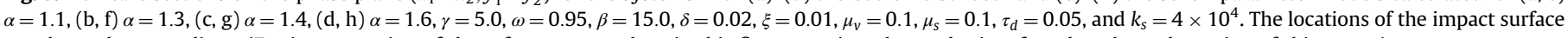
are shown by green lines. (For interpretation of the references to colour in this figure caption, the reader is referred to the web version of this paper.)

(a)

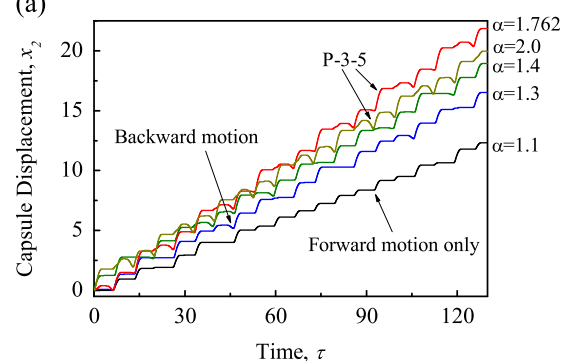

(b)

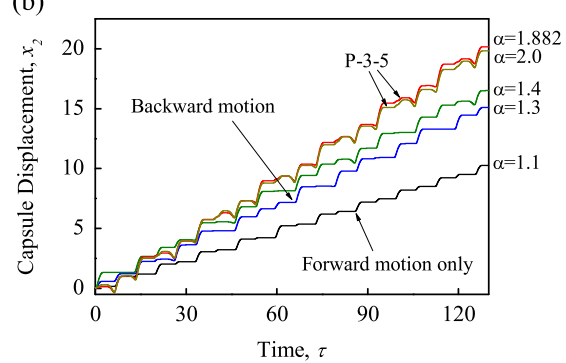

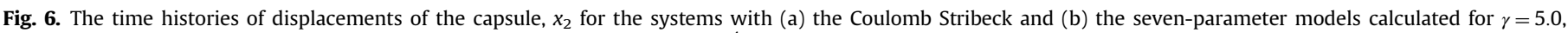

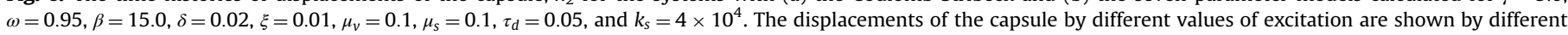
colours. (For interpretation of the references to colour in this figure caption, the reader is referred to the web version of this paper.) 


\subsection{Influence of damping ratio}

Fig. 8 presents a series of bifurcation diagrams which shows the evolution of the main attractors of the capsule system with the Coulomb model under variation of damping ratio $\xi$. As can be seen from Fig. 8(a), the chaotic motion of the capsule system is observed for $\alpha \in[0.132,0.928)$ when the damping ratio is 0.01 . When the damping ratio is 0.02 , the chaotic motion is observed for $\alpha \in[0.01,0.452]$ and $\alpha \in[0.686,0.796]$ as shown in Fig. 8(b). As the damping ratio increases, the region of the chaotic motion decreases as seen in Fig. 8(c) until it

(a)

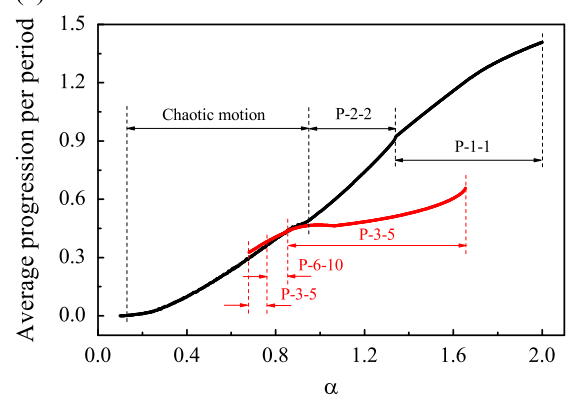

(c)

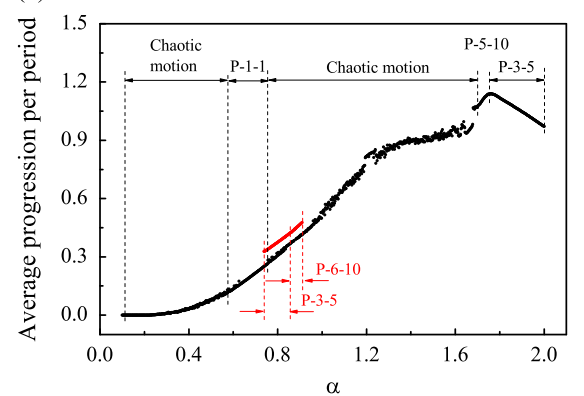

ceases to exist in Fig. 8(d). The evolution of the chaotic motion indicates that the behaviour of the capsule system becomes less complicated as its damping ratio increases which means that this parameter could be tuned in the system design for avoiding chaotic motion.

\section{Influence of excitation frequency}

This section investigates the influence of frequency of excitation on capsule dynamics and average progression using the bifurcation

(b)

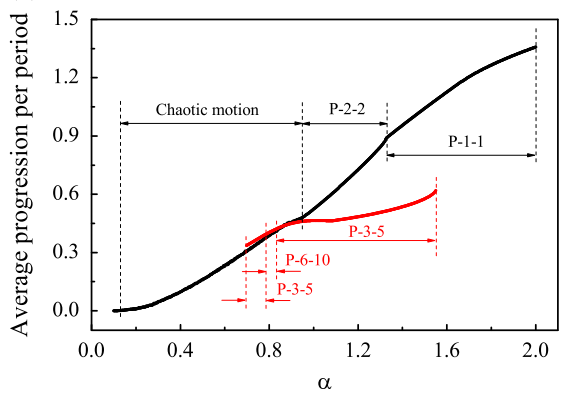

(d)

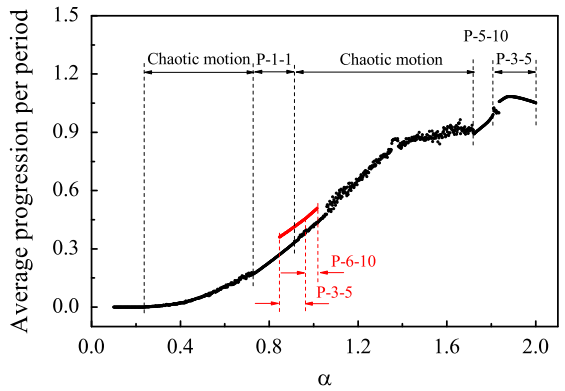

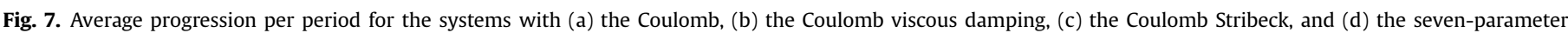
models calculated for $\gamma=5.0, \omega=0.95, \beta=15.0, \delta=0.02, \xi=0.01, \mu_{v}=0.1, \mu_{\mathrm{s}}=0.1, \tau_{d}=0.05$, and $k_{\mathrm{s}}=4 \times 10^{4}$. Average progressions of coexisting attractors are shown by red dots. (For interpretation of the references to colour in this figure caption, the reader is referred to the web version of this paper.)
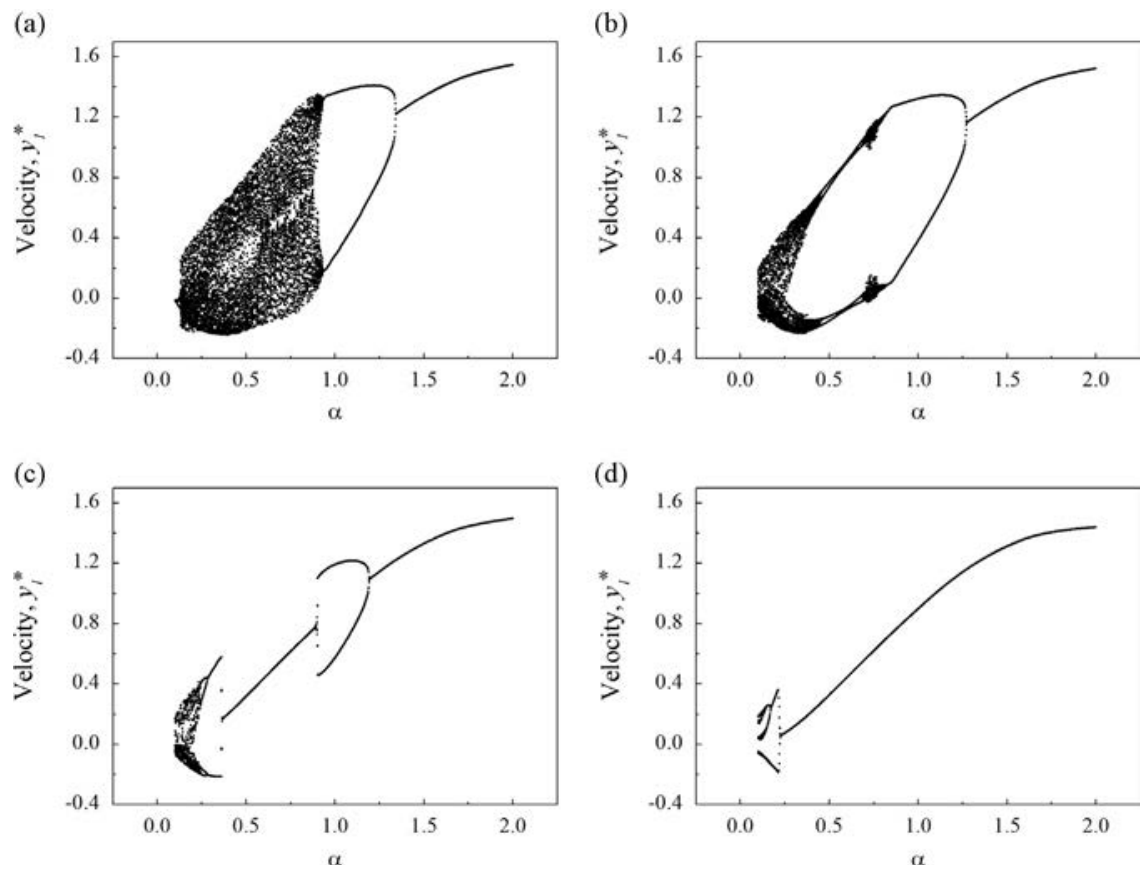

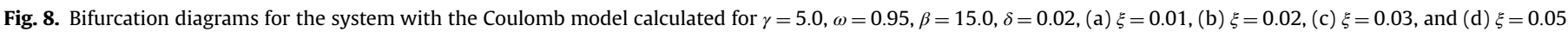

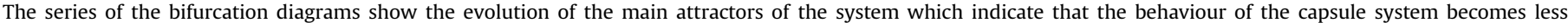
complicated as the damping ratio increases. 
diagrams with the four friction models as shown in Fig. 9. The main attractors are marked by black dots and the coexisting attractors are shown by red dots. Here we are interested in the global and local optima of the frequencies of excitation which give the capsule the best progression within the range of $\omega \in[0.8,1.2]$. The main idea of control of excitation frequency is to operate the capsule system within the range of global (or local) optimum which gives the fastest global (or local) average speed of the capsule progression.

\subsection{Bifurcation analysis}

It can be seen from Fig. 9 that the capsule system has very similar behaviour for all four friction models under varying the

(a)

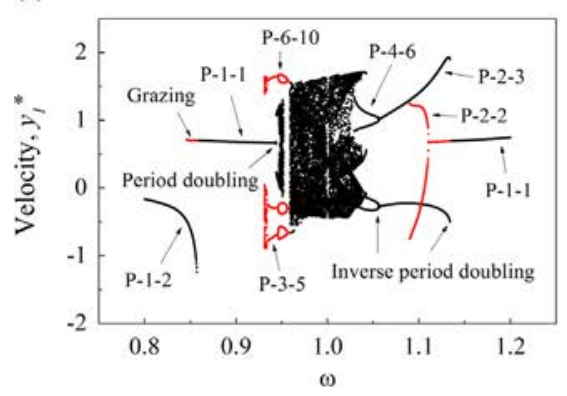

(b)

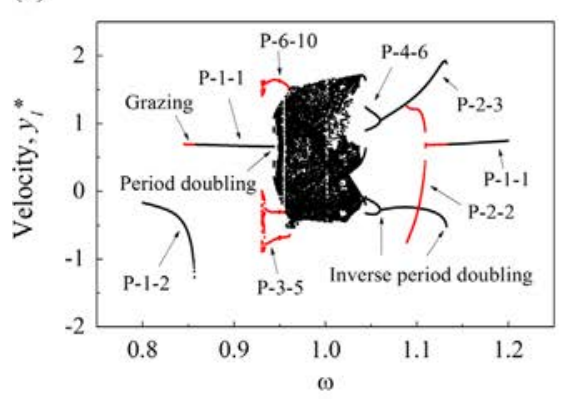

(c)

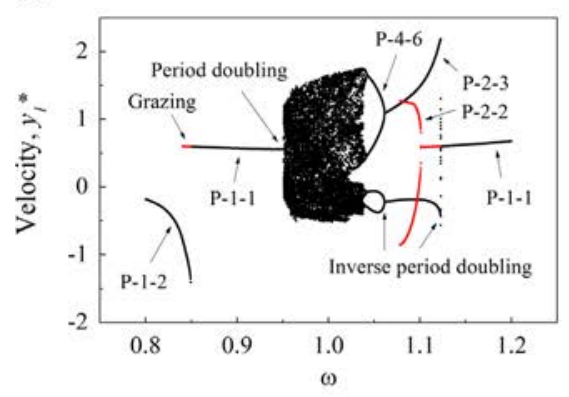

(d)

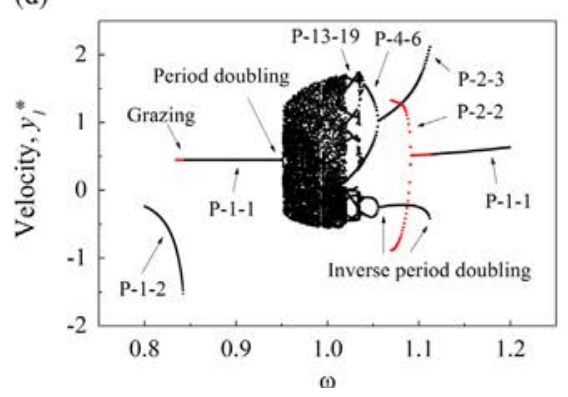

frequency of excitation. As the frequency increases, the capsule system bifurcates from a period- 1 motion with two impacts into a period-1 motion with one impact through a grazing bifurcation followed by a period doubling cascade leading to chaos. Then the chaotic motion bifurcates into a period- 1 motion with one impact through an inverse period doubling cascade.

Coexisting attractors are recorded for $\omega \in[0.9312,0.959]$ and $\omega \in[0.9304,0.9612]$ for the capsule system with the Coulomb and the Coulomb viscous damping models, respectively. Both of them are observed as a period-3 motion with five impacts, which bifurcates into a period- 6 motion with ten impacts followed by a period-3 motion with five impacts as the frequency of excitation decreases, and eventually through period doubling cascade leads
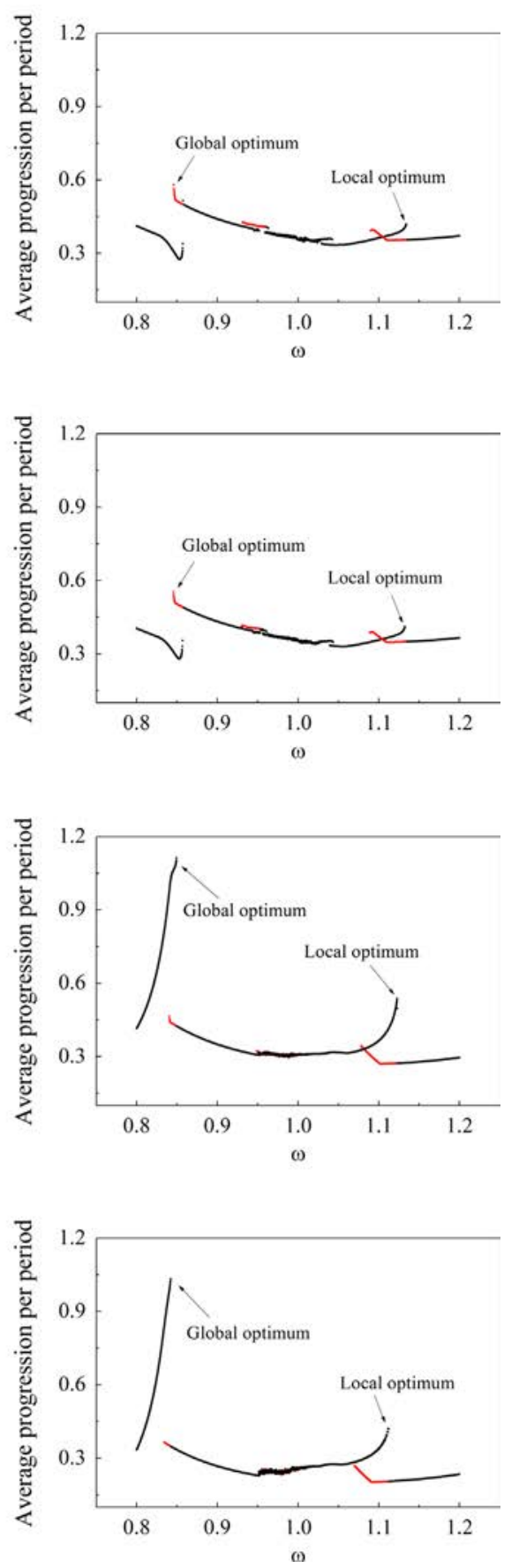

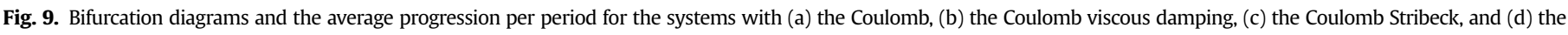

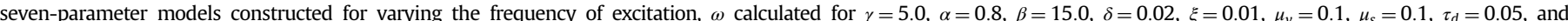

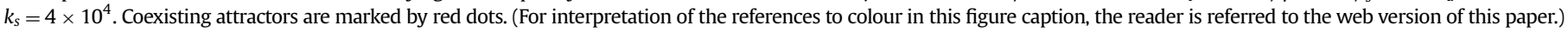


to the chaotic motion. However these coexisting attractors do not have significantly higher average progression of the system.

\subsection{Global and local optima}

Coexisting attractors are recorded for the system with the four friction models at where grazing bifurcation is encountered. Fig. 10 shows the time histories of attractors shown in red in Fig. 9 for $\omega=0.846$ where grazing is observed at the second impact of each period. It is worth noting that Fig. 10(a) and (b) depicts the global optimum frequency of excitation for the systems with the Coulomb and the Coulomb viscous damping models which gives the fastest average progression of the system. However the coexisting global optima for the systems with the Coulomb and the Coulomb viscous damping models are difficult to be obtained due to their very limited basins of attraction as shown in Fig. 11.

Fig. 12 shows the time histories of displacements of the systems with the four friction models on the main attractors at $\omega=0.842$. It is seen from the phase planes that the systems have period-1 motion with two impacts, but backward motions are observed in
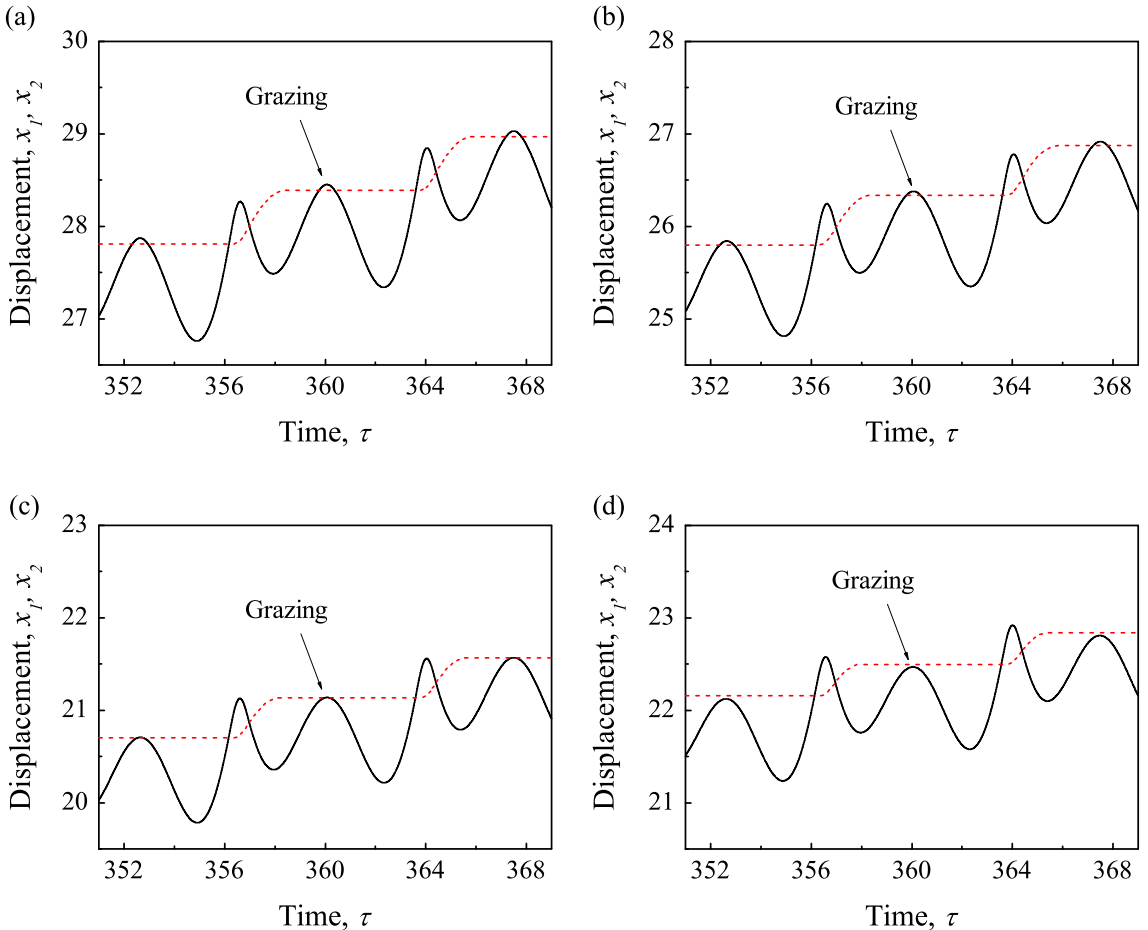

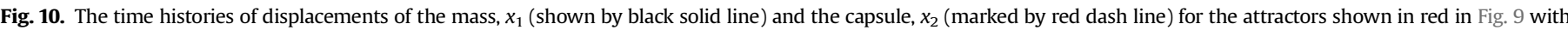

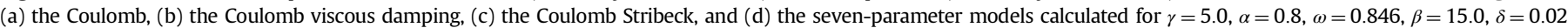

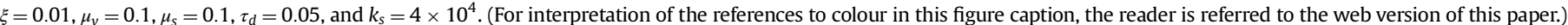

(a)

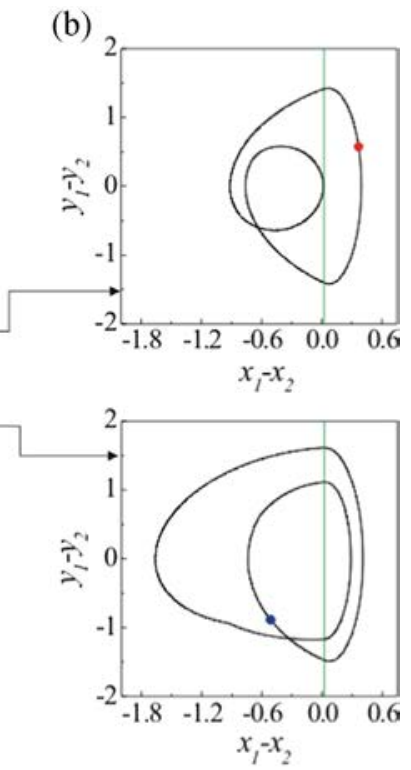

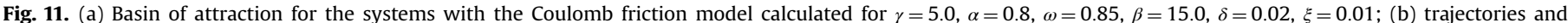
Poincaré maps for the coexisting period-1 with one impact and period-1 with two impacts solutions. 
Fig. 12(a) and (b) for the systems with the Coulomb and the Coulomb viscous damping models, so smaller average progression of the system is recorded. For the Coulomb Stribeck and the sevenparameter models, the global optimum frequencies of excitation are obtained because of their larger thresholds. To some extent, this scenario somehow demonstrates that friction may help to stabilize system and to improve capsule progression.
Local optima are recorded for the systems with the four friction models just before their inverse period doubling from the period-2 motion with three impacts to the period-1 motion with one impact. Fig. 13 demonstrates their local optima at $\omega=1.124$ where backward motion of the capsule with the Coulomb and the Coulomb viscous damping models is observed. In these cases the capsule progression in Fig. 13(a) and (c) does not have significant
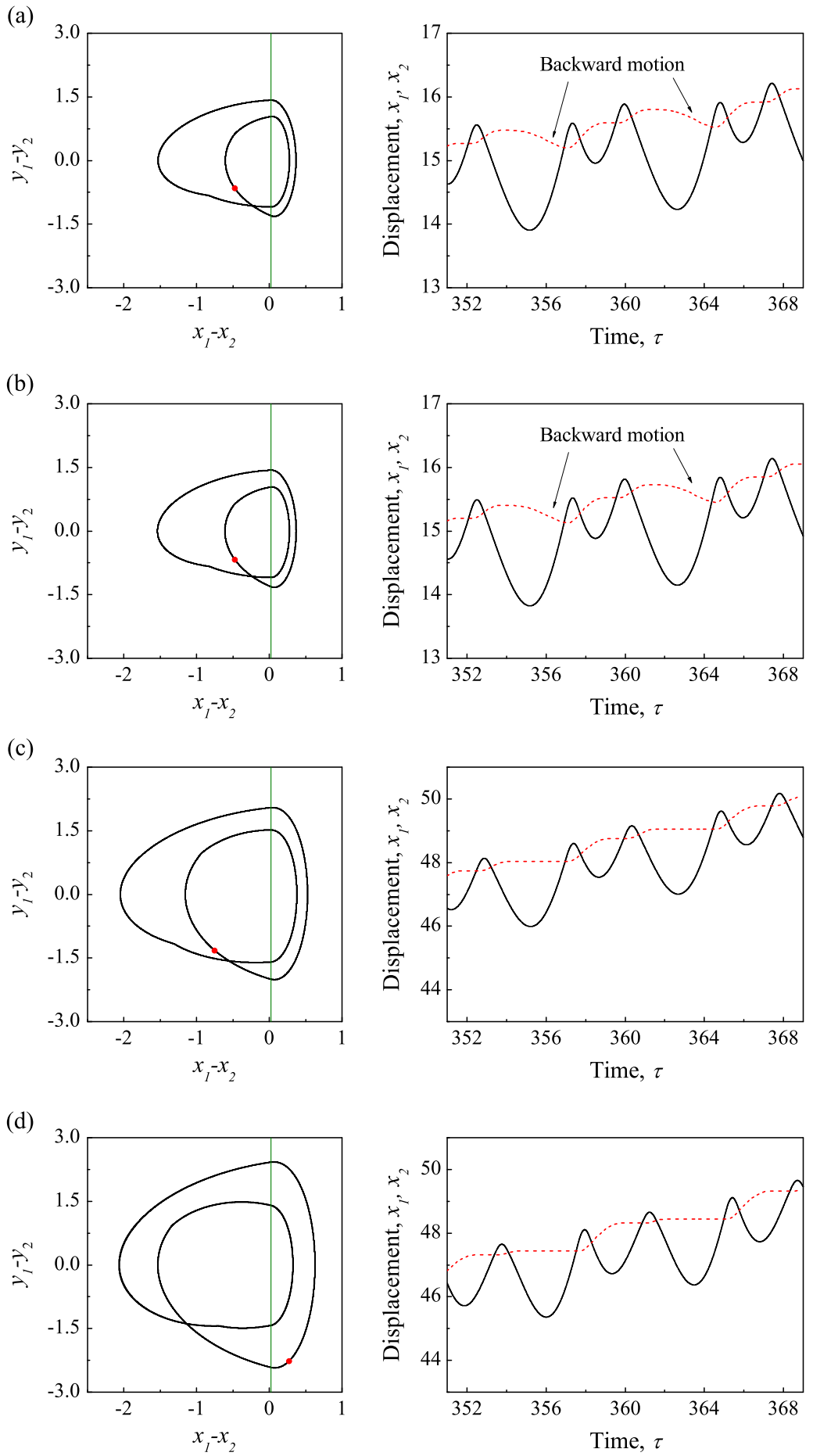

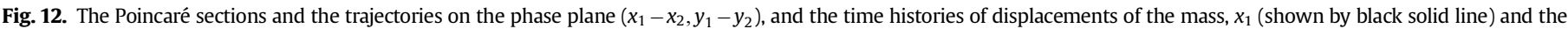

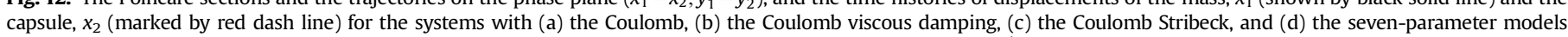

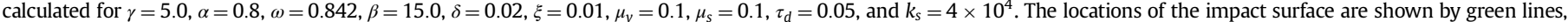
and Poincare sections are marked by red dots. (For interpretation of the references to colour in this figure caption, the reader is referred to the web version of this paper.) 
improvement in comparison with the progression obtained for their coexisting attractors in Fig. 13(b) and (d).

\section{Position feedback control}

\subsection{Control point}

In this section our proposed feedback control law will be applied to the control point which is defined as a $\gamma$ value where the system has no average progression but only oscillation, and then we will also show that our control method is applicable for chaos control and control of coexisting attractors. Fig. 14(a) shows the average progression of the capsule per period of external excitation, and its control point is observed at $\gamma=0.235$. Fig. 14(b) and (c) presents the trajectory on the phase plane $\left(x_{1}-x_{2}, y_{1}-y_{2}\right)$ and the time history of displacements of the mass, $x_{1}$ and the capsule, $x_{2}$ obtained for the control point, respectively. As can be seen from Fig. 14(c), the mass

(a)
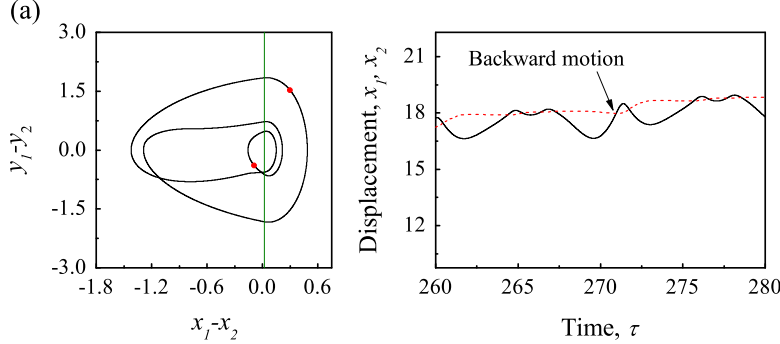

(c)
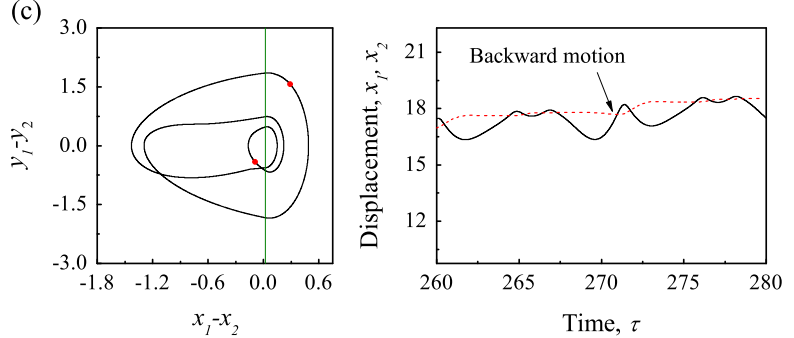

(d)
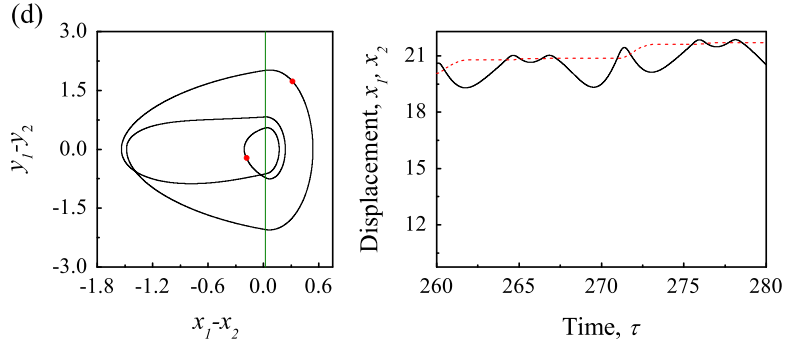

(f)
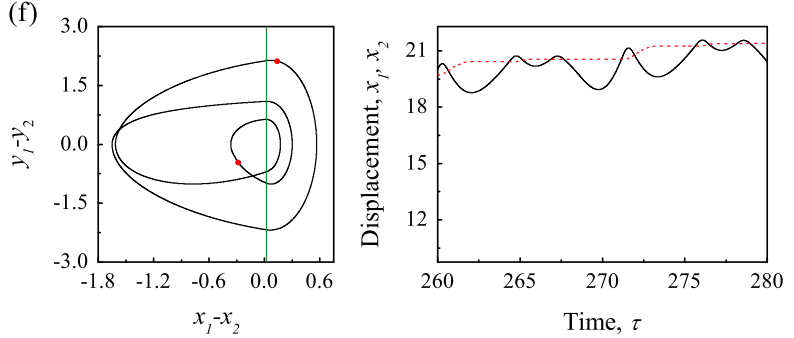

and the capsule oscillate around their origins which leads to no average progression for the entire system.

\subsection{Position feedback control}

Consider the position feedback control law

$u=k_{p}\left|x_{2}-x_{1}\right|$,

where $k_{p}$ is a linear position control gain. The resulting equation of motion of the capsule system is described by

$$
\begin{aligned}
\dot{x}_{1}= & y_{1}, \\
\dot{y}_{1}= & \alpha \cos (\omega \tau)+f_{p}\left(x_{1}, x_{2}\right)+2 \xi\left(y_{2}-y_{1}\right)-H_{3} \beta\left(x_{1}-x_{2}-\delta\right), \\
\dot{x}_{2}= & y_{2}\left(H_{1}\left(1-H_{3}\right)+H_{2} H_{3}\right), \\
\dot{y}_{2}= & \left(H_{1}\left(1-H_{3}\right)+H_{2} H_{3}\right)\left(-f_{s}-\left(x_{2}-x_{1}\right)-2 \xi\left(y_{2}-y_{1}\right)\right. \\
& \left.+H_{3} \beta\left(x_{1}-x_{2}-\delta\right)\right) / \gamma,
\end{aligned}
$$

(b)
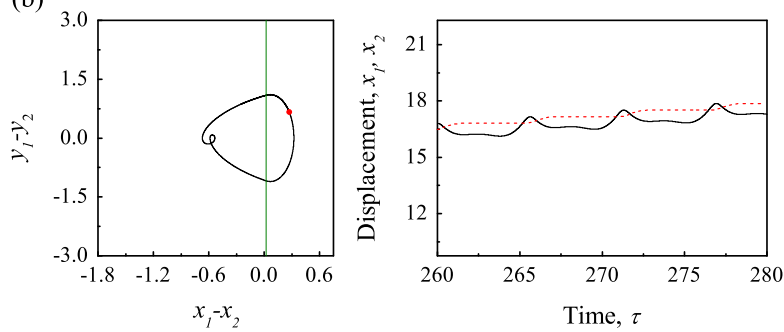

(d)
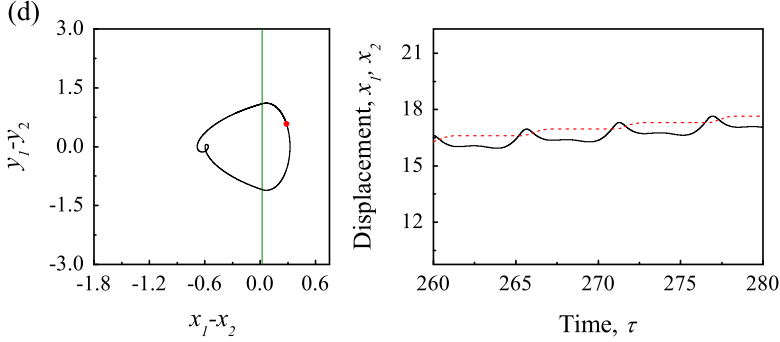

(e)
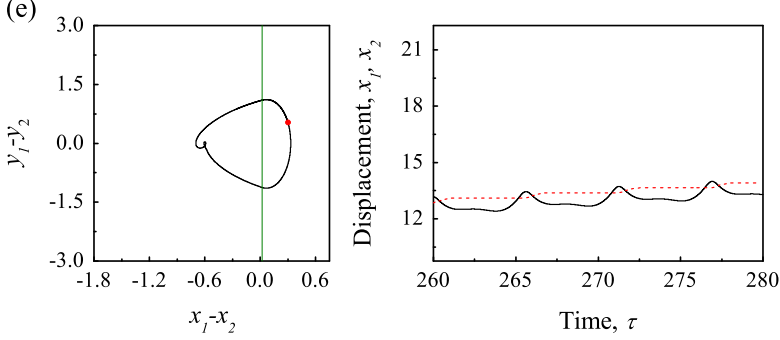

(g)
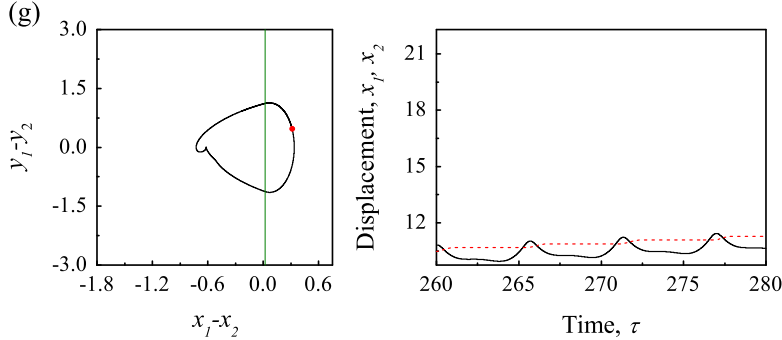

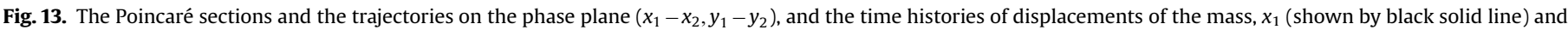

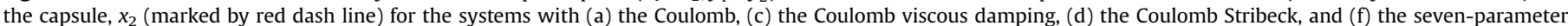

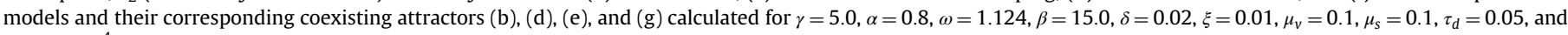

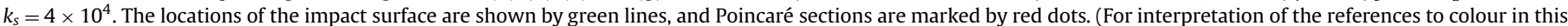
figure caption, the reader is referred to the web version of this paper.) 


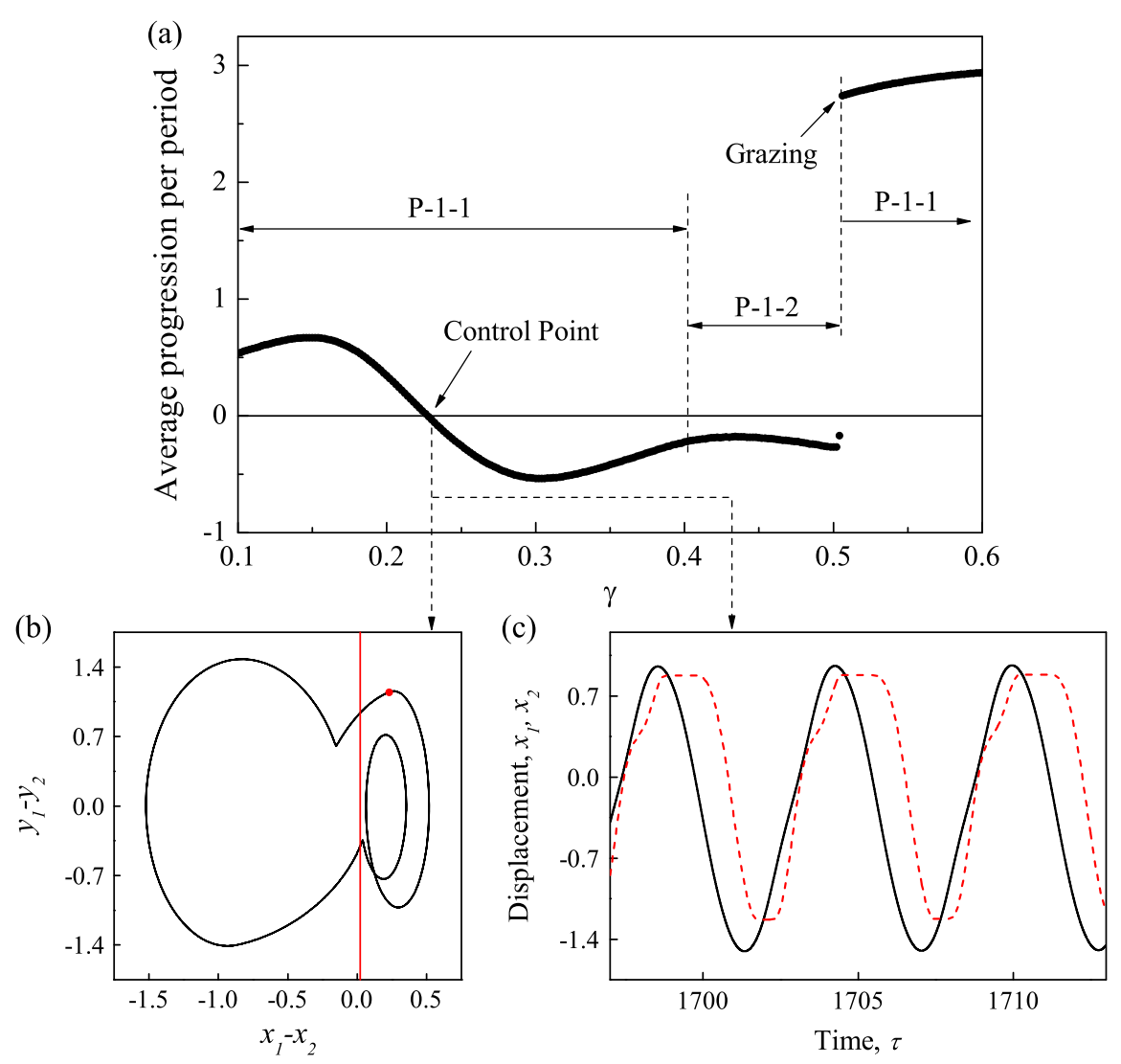

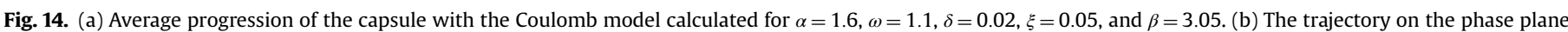

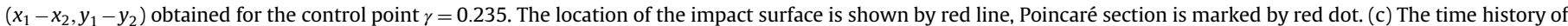

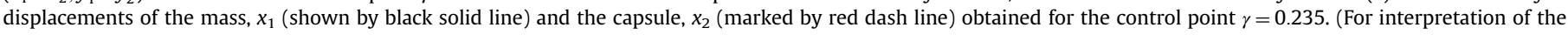
references to colour in this figure caption, the reader is referred to the web version of this paper.)

where

$f_{p}\left(x_{1}, x_{2}\right)=k_{p}\left|x_{2}-x_{1}\right|+\left(x_{2}-x_{1}\right)= \begin{cases}\left(1-k_{p}\right)\left(x_{2}-x_{1}\right) & \text { if } x_{1} \geq x_{2}, \\ \left(1+k_{p}\right)\left(x_{2}-x_{1}\right) & \text { if } x_{1}<x_{2}\end{cases}$

As we have found in [11], the optimal parameters of the capsule system for the best progression and for the minimum energy consumption are different. Here we use the control efficiency which is the ratio of the capsule progression per period of the external excitation, $T$ to the work done by the external force and the controller over one period

$E_{\text {avg }}=\frac{x_{2}(T)-x_{2}(0)}{\int_{0}^{T}[\alpha \cos (\omega \tau)+u(\tau)] \cdot y_{1}(\tau) \mathrm{d} \tau}$

in order to take the energy consumption into account for evaluating our proposed position feedback control law.

Fig. 15(a) presents the bifurcation diagram where the position control gain $k_{p}$ is used as a branching parameter. Additional windows in Fig. 15(a) show the trajectories on the phase plane, where the relative displacement $\left(x_{1}-x_{2}\right)$ is given on the horizontal axis, and the relative velocity $\left(y_{1}-y_{2}\right)$ is on the vertical axis. It can be observed that the system response is period-one motion for all the values of the position control gain for $k_{p} \in[-0.2,0.2]$, and the system behaviour changes from period-one motion with one impact per period of excitation to period-one with two impacts when the control gain varies from negative to positive. The average progression of the system as a function of the control gain $k_{p}$ is shown in Fig. 15(b), where the bidirectional motion of the system is depicted. Additional windows show the time history of displacements of the mass and the capsule, where we notice that negative control gain leads to the backward motion of the capsule, while positive control gain leads to forward motion.

\subsection{Chaos control}

Fig. 16 demonstrates the chaotic motion of the system with the Coulomb Stribeck model at $\alpha=1.3$ controlled by the position feedback control law when the control gain is chosen as $k_{p}=1.0$. Fig. 16(a) shows that the chaotic response of the system is changed to a period-2 response with four impacts per period of excitation when the control law is applied. Fig. 16(b) depicts the capsule displacements when the control action is switched on (marked by red line) and off (shown by grey line). It can be seen that despite the capsule speed is lower when the control is on, the capsule motion is periodic and forward only. Fig. 16(c) shows that no negative friction is recorded when the control action is switched on which confirms again that the capsule only has forward motion. The external excitations for the capsule system with and without applying the position feedback control action are depicted in Fig. 17(a) by using red and grey lines respectively, which shows much smaller amplitude of excitation with control than the one without control. Fig. 17(b) indicates that the capsule system without control has lower control efficiency than the one with control.

\subsection{Control of coexisting attractors}

The position feedback control law proposed here is not only used for chaos control, but also for control of coexisting attractors for the capsule system. Here we use the capsule system with the Coulomb model whose coexisting attractors are observed for $\alpha \in[0.682,1.654]$ as an example. The evolution of the basins of attraction computed for the range where coexisting attractors exist 


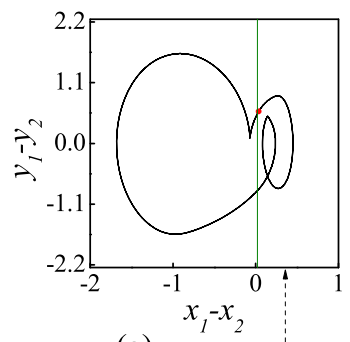

(a)
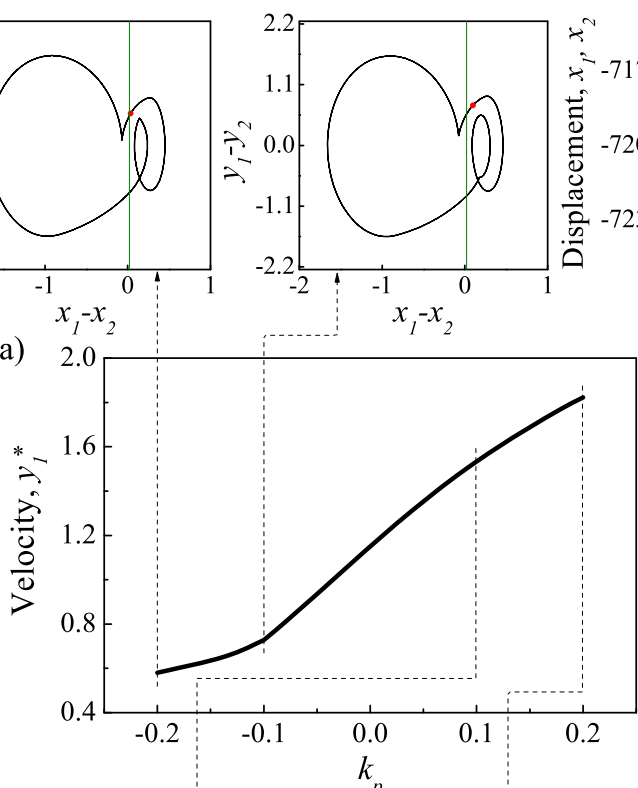
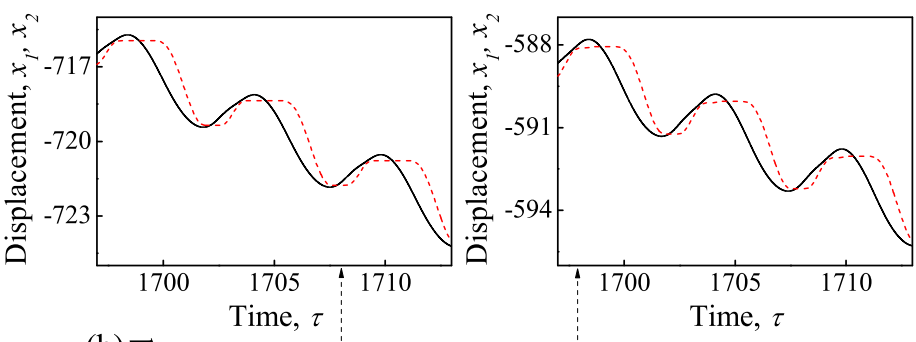

(b)
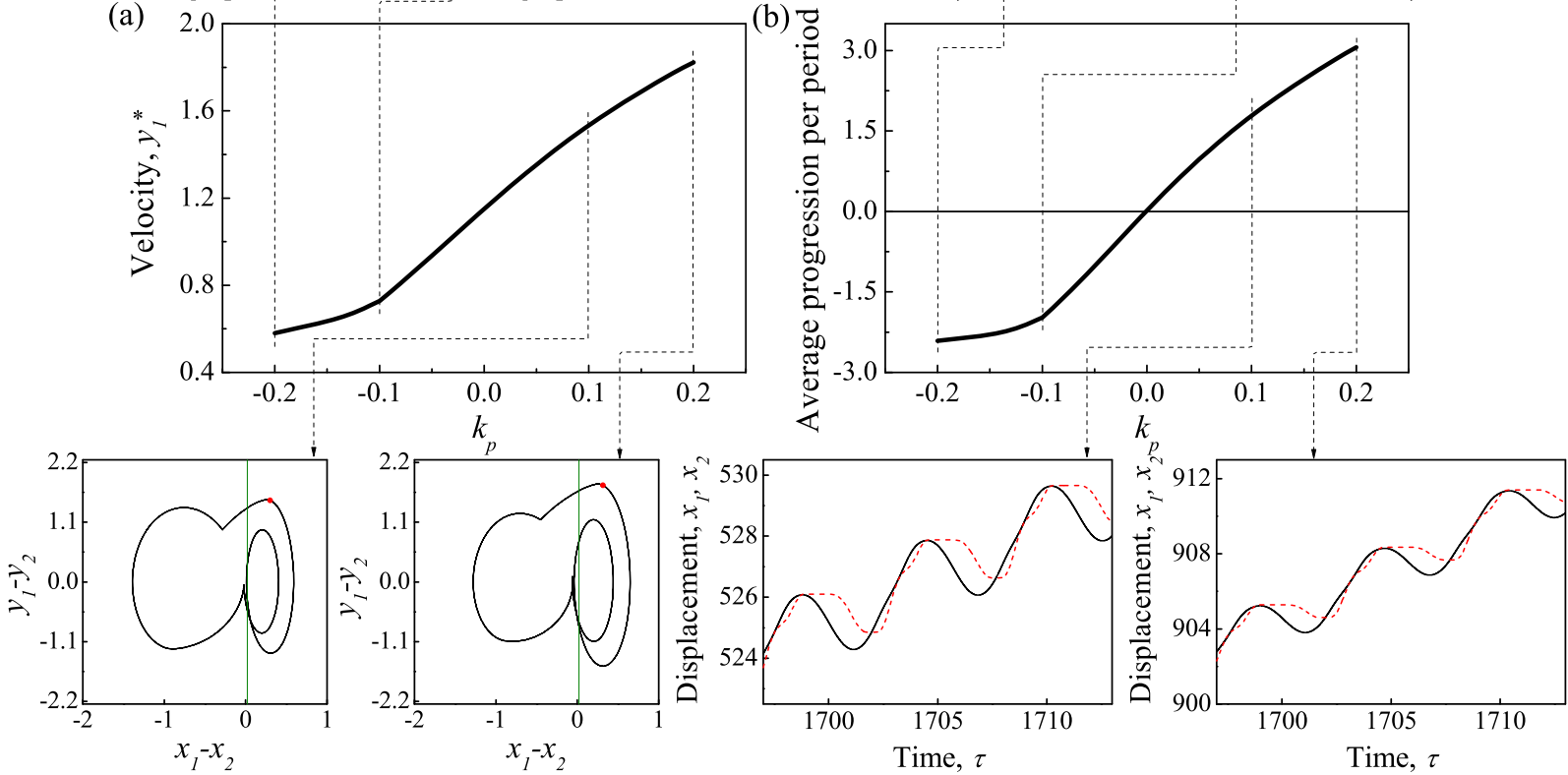

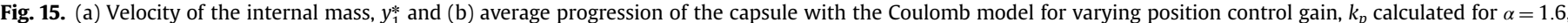

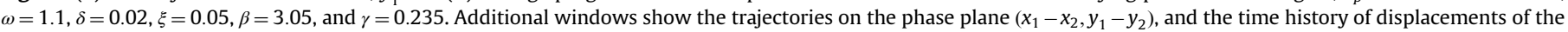

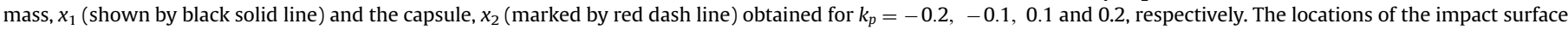

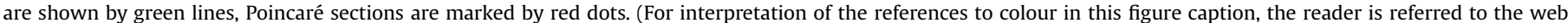
version of this paper.)

(a)

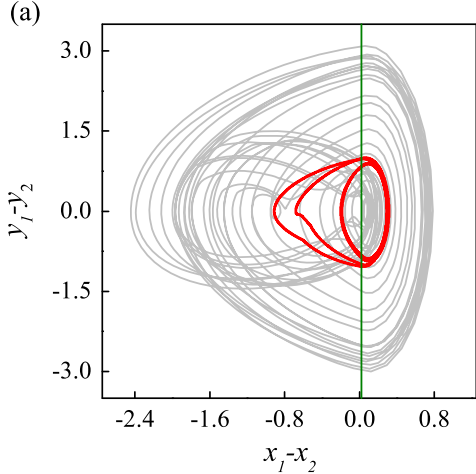

(b)

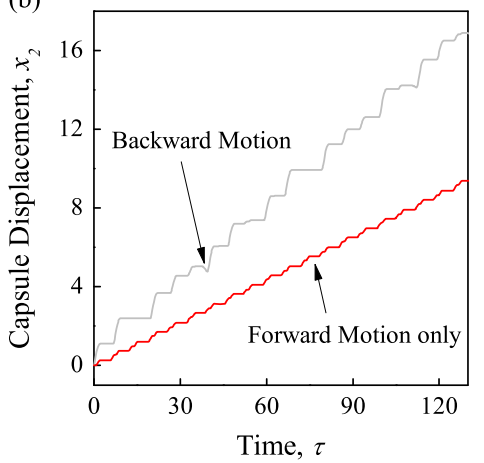

(c)

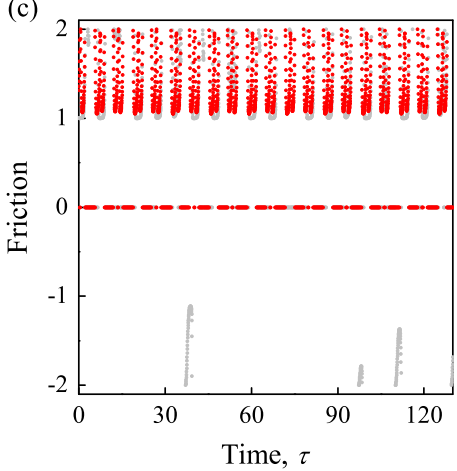

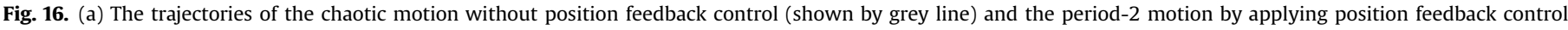

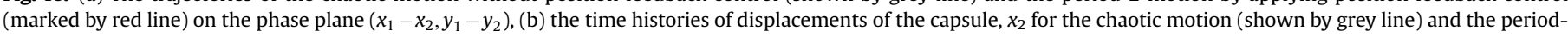

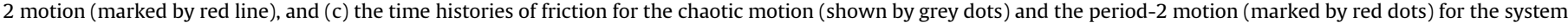

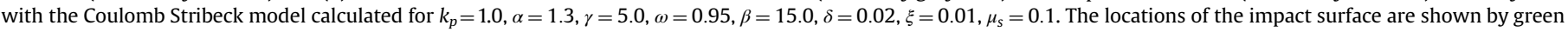
lines. (For interpretation of the references to colour in this figure caption, the reader is referred to the web version of this paper.)

is presented in Fig. 18. As can be seen from this figure, the dominant chaotic attractor is marked by green dots, and its basin is given in yellow. The period-3 attractor marked by black dots emerges at $\alpha=0.682$ in Fig. 18(a), and bifurcates into a period-6 attractor as shown in Fig. 18(b). The chaotic attractor is turned into a period-2 attractor in Fig. 18(c) while its basin is gradually shrinking when the amplitude of excitation increases. The period- 6 attractor becomes a period-3 attractor again in Fig. 18(c) whose basin shrinks from Fig. 18(d) to Fig. 18(f) until disappearing completely at $\alpha=1.654$. On the other hand, the period-2 attractor bifurcates into a period-1 attractor as seen in Fig. 18(d) to (f), and its basin is expanded gradually.

The evolution of the basins of attraction for the system with control at $\alpha=1.5$ is shown in Fig. 19. As can be seen in Fig. 19(a), the basin area for the period-3 attractor is approximately equivalent to the one for the dominant period- 1 attractor when the control action is not applied. Once the control is on for $k_{p}=0.05$ in Fig. 19(b), the period- 3 orbit is turned into a period- 6 orbit whose basin is shrunken. Then the period- 6 orbit moves to the period- 3 orbit again at $k_{p}=0.1$ in Fig. 19(c) at where its basin remains very small and 
(a)

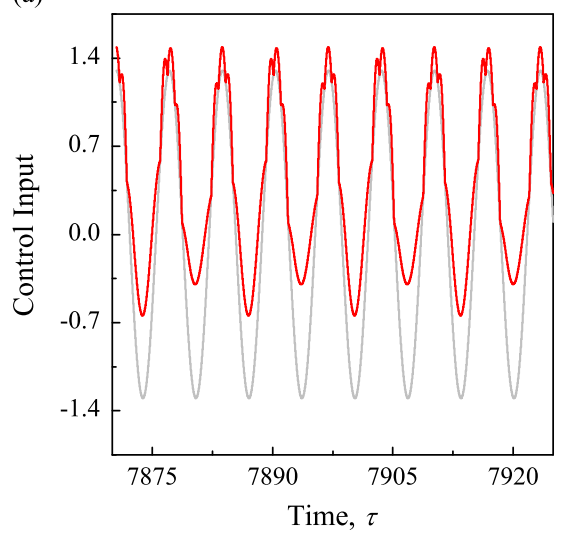

(b)

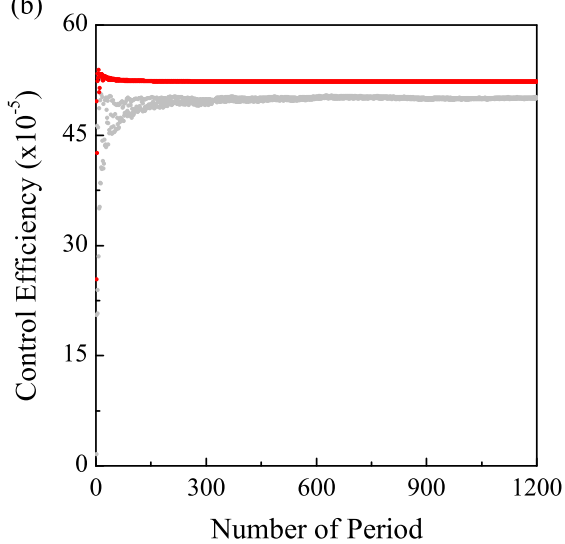

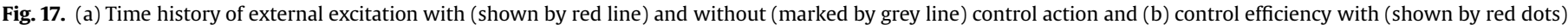

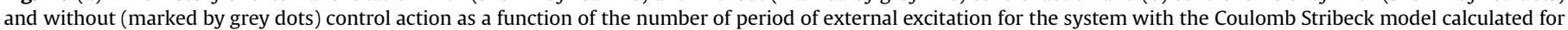

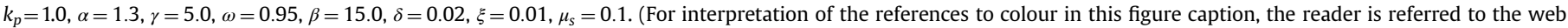
version of this paper.)

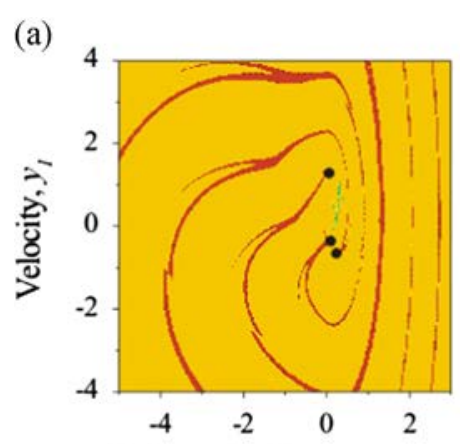

Relative displacement, $x_{1}-x_{2}$, $\alpha=0.682$

(d)

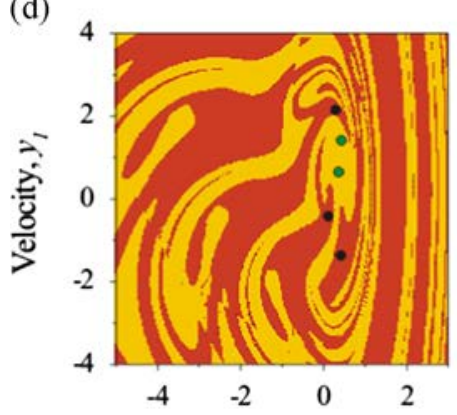

Relative displacement, $x_{1}-x_{2}$

$$
\alpha=1.2
$$

(b)

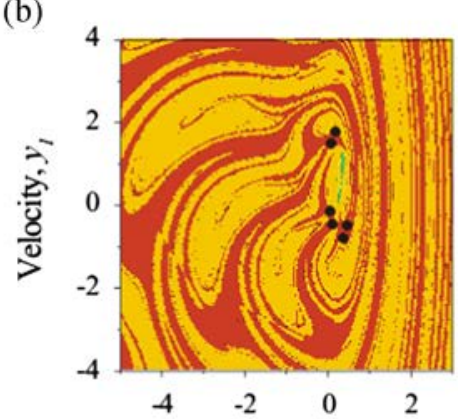

Relative displacement, $x_{1}-x$, $\alpha=0.8$

(e)

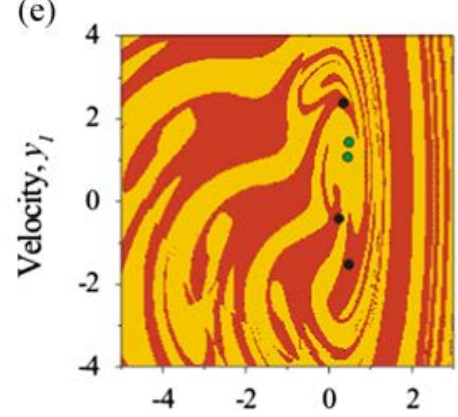

Relative displacement, $x_{1}-x_{2}$, $\alpha=1.338$

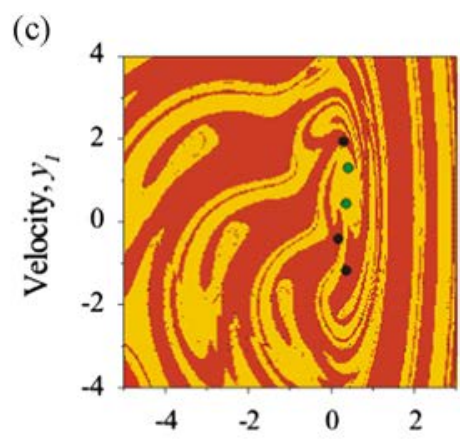

Relative displacement, $x_{1}-x_{2}$ $\alpha=1.07$

(f)

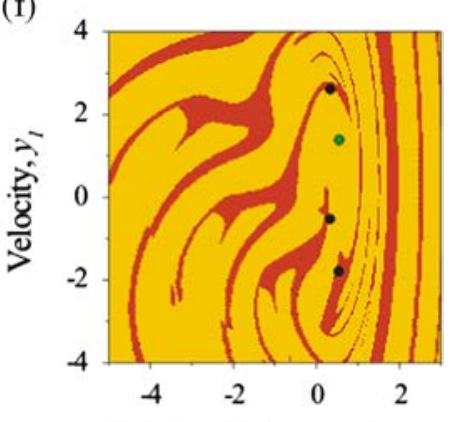

Relative displacement, $x_{1}-x_{2}$ $\alpha=1.6$

\section{Colour of basin}

\section{Colour of attractor}

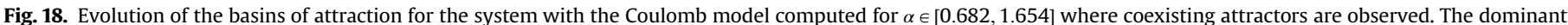

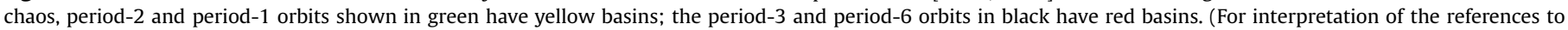
colour in this figure caption, the reader is referred to the web version of this paper.)

fractal while the basin for the dominant period-1 orbit is expanded significantly. The result reveals that, the basin of the dominant period- 1 orbit can be expanded by adjusting the control gain $k_{p}$, thus improving the probability of the control from a current attractor to a desired one.

Fig. 20 demonstrates the control from a period-3 motion to a desired period-1 motion by applying the position feedback control law for one period. Fig. 20(a) shows the projection of the Poincare map on the $y_{1}$-axis as a function of the number of period of external excitation. It can be seen that the control is applied at the period $n=300$ and the system is stabilized into the period-1 motion after a transient behaviour. The trajectories of the period-3 motion with five impacts before control and the desired period-1 motion with one impact after control are presented in 
(a)

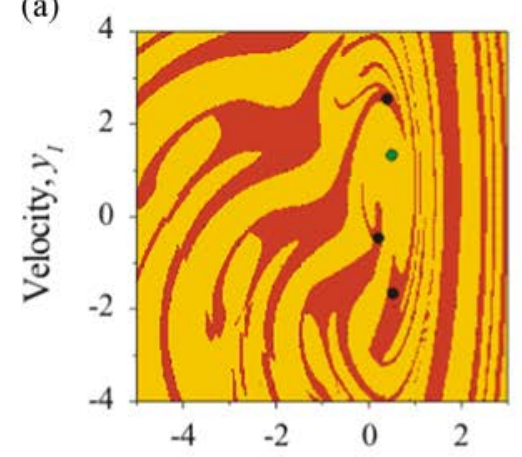

Relative displacement, $x_{1}-x_{2}$

(b)

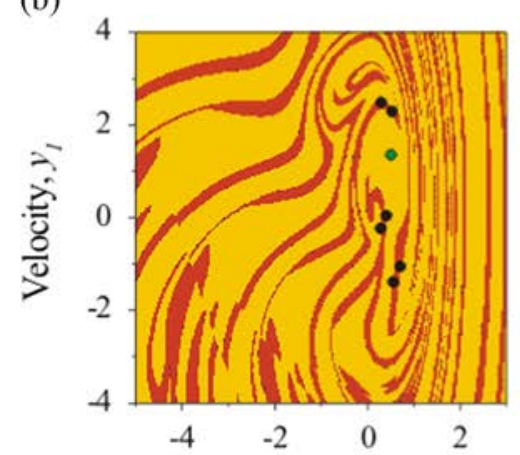

Relative displacement, $x_{1}-x_{2}$
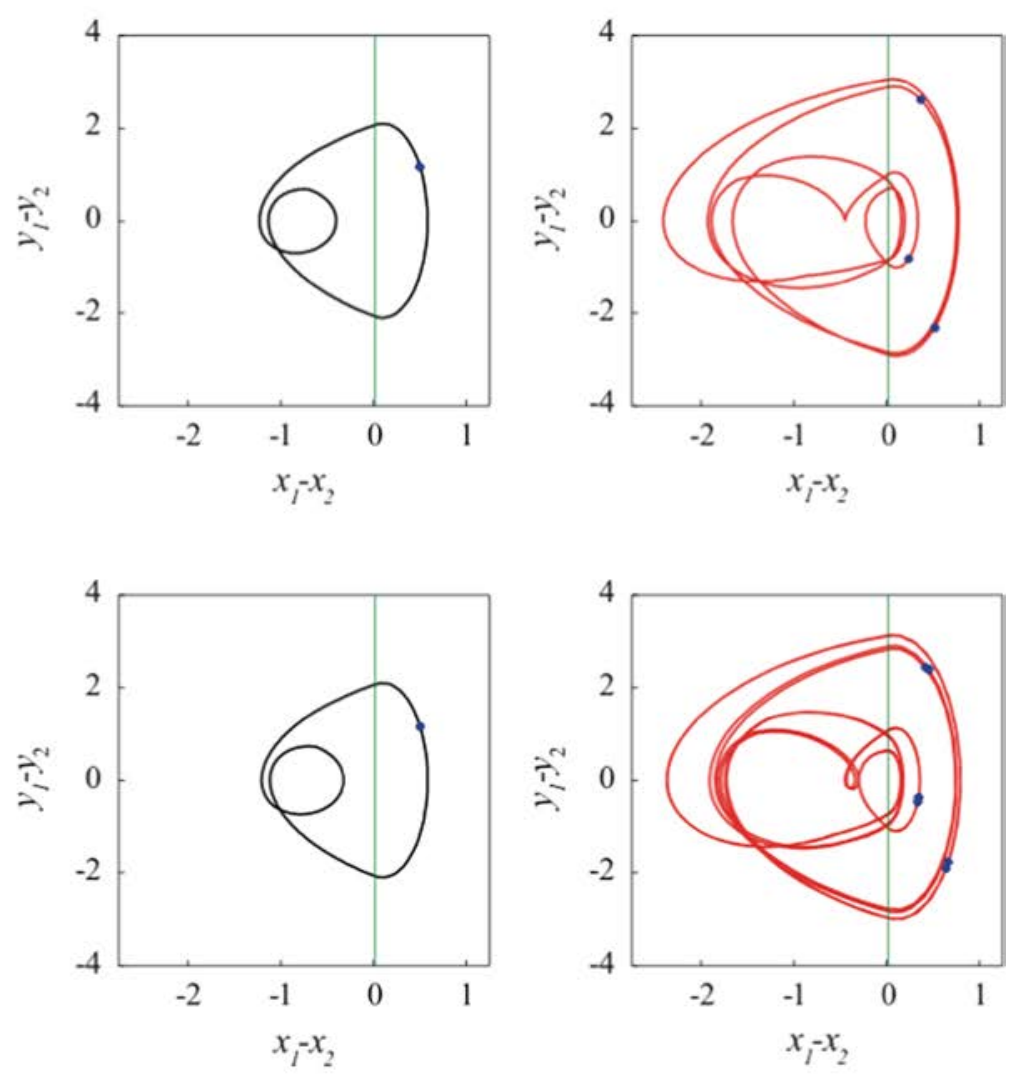

(c)

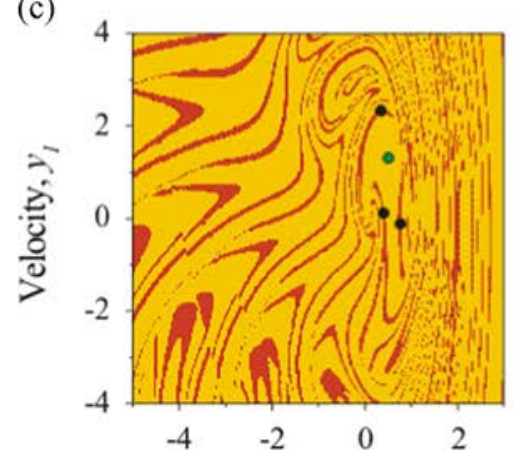

Relative displacement, $x_{1}-x_{2}$
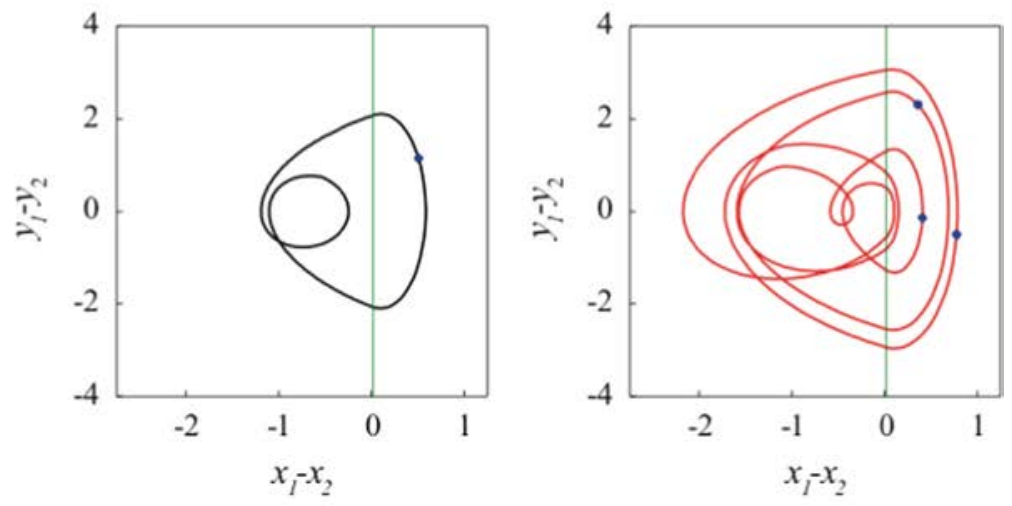

\section{Colour of basin}

\section{Colour of attractor}

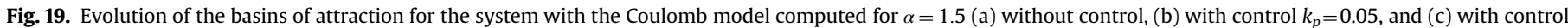

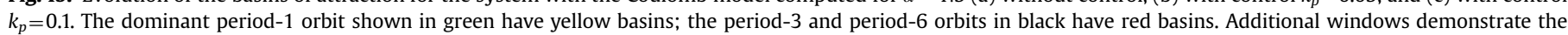

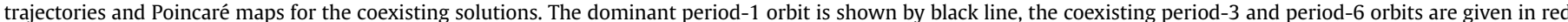

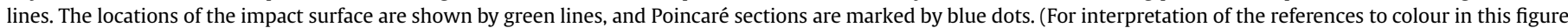
caption, the reader is referred to the web version of this paper.)

Fig. 20(b). Fig. 20(c) confirms the control action again by showing the time history of displacements of the mass and the capsule.

\section{Concluding remarks}

A capsule system driven by a harmonic force applied to its inner mass was considered in this study. Four various friction models were employed to describe motion of the capsule in different environments taking into account Coulomb friction, viscous damping, Stribeck effect, pre-sliding, and frictional memory. The non-linear dynamics analysis was conducted to identify the optimal amplitude and frequency of the applied force in order to achieve the motion in the required direction and to maximize its speed.

The variation of the amplitude of external excitation was considered in the range $\alpha \in[0.1,2.0]$ for the mass ratio of $\gamma=5.0$ and the stiffness ratio of $\beta=15.0$. Our studies revealed that for amplitudes of the harmonic force smaller than the threshold of friction, the dynamic behaviour of the system was mainly chaotic. Once the amplitude of the harmonic force overcomes this limit, the motion of 
(a)

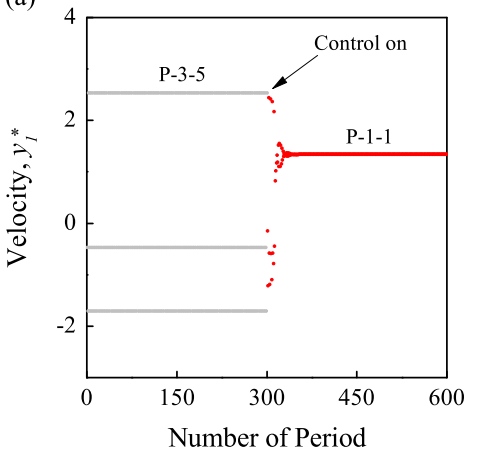

(b)

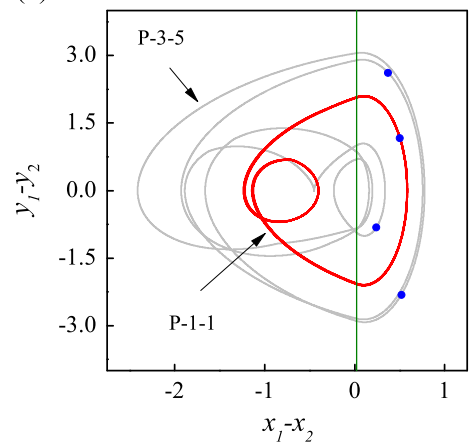

(c)

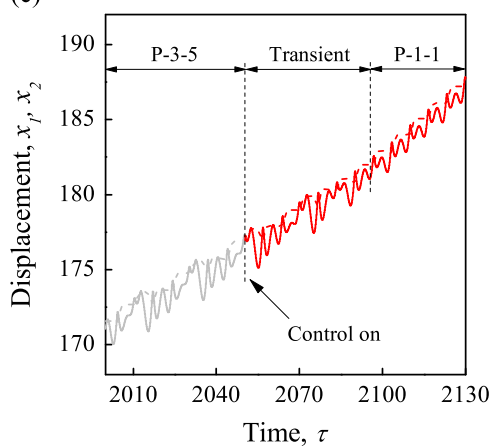

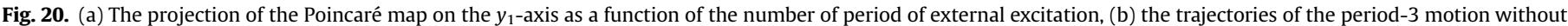

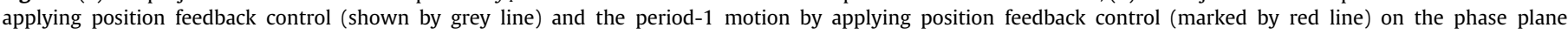

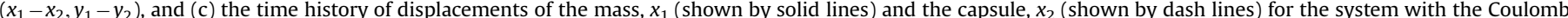

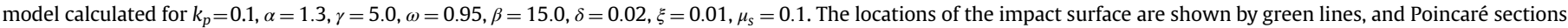
are marked by blue dots. (For interpretation of the references to colour in this figure caption, the reader is referred to the web version of this paper.)

the capsule system becomes periodic. It was also obtained that when the amplitude of the harmonic force was close to the threshold of friction force, the period-3 responses obtained for the systems with the Coulomb Stribeck and the seven-parameter models were very close to the coexisting period-3 responses recorded for the systems with the Coulomb and the Coulomb viscous damping models. This means that similar behaviour of the capsule system can be expected for the amplitude of the harmonic force close to the threshold of friction in all friction conditions.

The excitation frequency was varied in the range $\omega \in[0.8,1.2]$. Here the capsule system with all four friction models has similar bifurcation structure. As the frequency increases, the capsule system bifurcates from periodic motion to chaos through a period doubling cascade, and then bifurcates into periodic motion via an inverse period doubling cascade. Global and local optima which gave the fastest speed of the system were observed. It is noted that the global optima for the systems with the Coulomb and the Coulomb viscous damping models are obtained for those of their co-existing attractors which have very limited basins of attraction. The global optima for the systems with the Coulomb Stribeck and the seven-parameter models belong to the attractors with large basins. The larger thresholds of frictional force in these models result in the stabilization of the system and improvement of capsule progression.

The variation of damping ratio has shown that the behaviour of the system becomes less complex as the damping ratio increases, which means that this parameter could be tuned in the system design for avoiding chaotic motion.

The position feedback control law was applied to the capsule system with the Coulomb friction model. With the control in place, the capsule system is able to move forward and backward under variation of its position control gain, $k_{p}$. When the value of this gain varies from negative to positive, the system response is transformed from period-one motion with one impact to period one with two impacts, while the direction of motion changes from backward to forward. Here the larger the control gain is, the faster the average progression of the capsule is.

To demonstrate the application of the proposed position feedback control law for chaos control, the system with Coulomb Stribeck model was considered at $\alpha=1.3$, where for the chosen system parameters the chaotic motion was observed. When the control law was applied, a period-2 response with four impacts per period of excitation was obtained. In this case the capsule speed was lower when the control was applied, but the capsule motion was periodic and forward only. Also the capsule with control has higher control efficiency than the one without control.
The evolution of the basins of attraction was also studied for the system with the Coulomb friction model for the range of excitation amplitude where a number of attractors coexist, and the effect of the control law on the evolution of the basins was investigated. The result showed that the basin of the desired attractor can be significantly enlarged by adjusting the control gain slightly. Therefore, a chance of switching from the current attractor to a desired one can be improved.

\section{Acknowledgements}

Dr Yang Liu would like to acknowledge the financial support for the Research Project of State Key Laboratory of Mechanical System and Vibration (MSV201401) by Shanghai Jiao Tong University and the Small Research Grant (31841) by the Carnegie Trust for the Universities of Scotland.

\section{References}

[1] F. Carpi, S. Galbiati, A. Carpi, Controlled navigation of endoscopic capsules: concept and preliminary experimental investigations, IEEE Trans. Biomed. Eng. 54 (2007) 2028-2036.

[2] P. Glass, E. Cheung, M. Sitti, A legged anchoring mechanism for capsule endoscopes using micropatterned adhesives, IEEE Trans. Biomed. Eng. 55 (2008) 2759-2767.

[3] B.J. Nelson, I.K. Kaliakatsos, J.J. Abbott, Microrobots for minimally invasive medicine, Annu. Rev. Biomed. Eng. 12 (2010) 55-85.

[4] G. Ciuti, A. Menciassi, P. Dario, Capsule endoscopy: from current achievements to open challenges, IEEE Trans. Biomed. Eng. 4 (2011) 59-72.

[5] Z. Wang, H. Gu, A bristle-based pipeline robot for I11-constraint pipes, IEEE Trans. Mechatron. 13 (2008) 383-392.

[6] Y. Zhang, S. Jiang, X. Zhang, X. Ruan, D. Guo, A variable-diameter capsule robot based on multiple wedge effects, IEEE/ASME Trans. Mechatron. 16 (2011) 241-254.

[7] J. Park, D. Hyun, W. Cho, T. Kin, H. Yang, Normal-force control for an in-pipe robot according to the inclination of pipelines, IEEE Trans. Ind. Eletron. 58 (2011) 5304-5310.

[8] H. Li, K. Furuta, F.L. Chernousko, Motion generation of the capsubot using internal force and static friction, in: Proceedings of the 45th IEEE Conference on Decision and Control, San Diego, CA, USA, 2006, pp. 6575-6580.

[9] F.L. Chernousko, The optimum periodic motions of a two-mass system in a resistant medium, J. Appl. Math. Mech. 72 (2008) 116-125.

[10] H.B. Fang, J. Xu, Dynamics of a mobile system with an internal accelerationcontrolled mass in a resistive medium, J. Sound Vib. 330 (2011) 4002-4018.

[11] Y. Liu, M. Wiercigroch, E. Pavlovskaia, H. Yu, Modelling of a vibro-impact capsule system, Int. J. Mech. Sci. 66 (2013) 2-11.

[12] S. Yim, M. Sitti, Design and rolling locomotion of a magnetically actuated soft capsule endoscope, IEEE Trans. Robot. 28 (2012) 183-194.

[13] L.J. Sliker, M.D. Kern, J.A. Schoen, M.E. Rentschler, Surgical evaluation of a novel tethered robotic capsule endoscope using micro-patterned treads, Surg. Endosc. 26 (2012) 2862-2869.

[14] Y. Liu, E. Pavlovskaia, M. Wiercigroch, Vibro-impact responses of capsule system with various friction models, Int. J. Mech. Sci. 72 (2013) 39-54. 
[15] E. Gutierrez, D.K. Arrowsmith, Control of a double impacting mechanical oscillator using displacement feedback, Int. J. Bifurc. Chaos 14 (2004) 3095-3113.

[16] H. Dankowicz, J. Jerrelind, Control of near-grazing dynamics in impact oscillators, Proc. R. Soc. A 461 (2005) 3365-3380.

[17] S.L.T. de Souza, I.L. Caldas, Controlling chaotic orbits in mechanical systems with impacts, Chaos Solitons Fractals 19 (2004) 171-178.

[18] J.Y. Lee, J.J. Yan, Control of impact oscillator, Chaos Solitons Fractals 28 (2006) $136-142$.

[19] S.L.T. de Souza, I.L. Caldas, R.L. Viana, Damping control law for a chaotic impact oscillator, Chaos Solitons Fractals 32 (2007) 745-750.

[20] L. Wang, X. Xu, Y. Li, Impulsive control of a class of vibro-impact systems, Phys. Lett. A 372 (2008) 5309-5313.

[21] Y. Liu, M. Wiercigroch, J. Ing, E. Pavlovskaia, Intermittent control of coexisting attractors, Philos. Trans. R. Soc. A 371 (2013) 20120428.
[22] J. Ing, E. Pavlovskaia, M. Wiercigroch, S. Banerjee, Bifurcation analysis of an impact oscillator with a one-sided elastic constraint near grazing, Physica D 239 (2010) 312-321.

[23] L.C. Silva, M.A. Savi, A. Paiva, Nonlinear dynamics of a rotordynamic nonsmooth shape memory alloy system, J. Sound Vib. 332 (2013) 608-621.

[24] E. Pavlovskaia, M. Wiercigroch, Periodic solution finder for an impact oscillator with a drift, J. Sound Vib. 267 (2003) 893-911.

[25] G.W. Luo, X.H. Lv, L. Ma, Periodic-impact motions and bifurcations in dynamics of a plastic impact oscillator with a frictional slider, Eur. J. Mech. A/Solids 27 (2008) 1088-1107.

[26] G.W. Luo, X.H. Lv, Controlling bifurcation and chaos of a plastic impact oscillator, Nonlinear Anal.: Real World Appl. 10 (2009) 2047-2061.

[27] J. Wojewoda, A. Stefański, M. Wiercigroch, T. Kapitaniak, Hysteretic effects of dry friction: modelling and experimental studies, Philos. Trans. R. Soc. A 366 (2008) 747-765 